Теоретичні та прикладні питання

\title{
Conspectus of old settlements flora of the Lower Dnipro
}

\author{
IVAN IVANOVYCH MOYSIYENKO \\ POLINA MYKHAILOVNA DAYNEKO \\ BARBARA SUDNIK-WÓJCIKOWSKA \\ IWONA DEMBICZ \\ MARIA ZACHWATOWICZ \\ MARINA YAROSLAVIVNA ZAKHAROVA
}

MOYSIYENKO I.I., DAYNEKO P.M., SUDNIK-WÓJCIKOWSKA B., DEMBICZ I., ZACHWATOWICZ M., ZAKHAROVA M.YA. (2020). Conspectus of old settlements flora of the Lower Dnipro. Chornomors'k. bot. z., 16 (1): 6-39. doi: 10.32999/ksu1990$553 \mathrm{X} / 2020-16-1-1$

During the past centuries high level of agricultural production resulted in a significant decrease steppe vegetation. In transformed landscapes South Ukraine cultural monuments such as old settlements can have the potential for biodiversity conservation. During the last decade (2010-2019) the flora of 18 earthworks of the Lower Dnipro ancient settlements with varied area from 1.1 to 18.7 ha was investigated. All settlements located on the steep bank of the Dnipro River on both sides (Kherson and Mykolayiv Regions), mainly between two closely spaced ravines or «balkas». The river terrace and ravines provided a natural protection for the settlements on three sides, and on the another side they were protected by artificial moat and shaft. The Dnipro was acknowledged as playing a key role in specific location of the earthworks as a leading waterway for trade and economic structure formation in the classical and Hellenistic periods. A list of the flora of vascular plants includes 526 species wich belongs to 279 genera, 74 families, 3 classes and 2 divisions. Total species richness ranged from 125 to 290 (178 species per archeological site in average). An appreciable number of native plants indicate a hight level of preservation of the objects. The contribution of native species in old settlements varied much from $62.1 \%$ (Liubymivske) to $81.1 \%$ (Velyke Tiagynske). The contribution of native species to the alien species is 3:1. Beside this, in the study area, we identified 30 species of protected vascular plants, of which 10 plant species are included in the Red Data Book of Ukraine (Astragalus borysthenicus, A. dasyanthus, Elytrigia stipifolia, Gymnospermium odessanum, Stipa capillata, S. lessingiana, S. pulcherrima, S. ucrainica, Tulipa biebersteiniana, $T$. gesneriana) and 20 plant species are included in the Red List of Kherson Province: Amygdalus nana, Bellevalia sarmatica, Bromopsis heterophylla, Dianthus andrzejowskianus, Ephedra distachya, Ferula capsica, Fraxinus excelsior, Hyacinthella leucophaea, Jurinea salicifolia, J. stochadifolia, Limonium platyphyllum, Linaria macroura, Linum czernjajevii, Prangos odontalgica, Quercus robur, Silene supina, Valeriana stolonifera, Veronica capsellicarpa, Vinca herbacea, Vitis sylvestris.

Keywords: vascular plants, rare communities, steppe, conservation of nature, Kherson region

МОЙСІєНКО І.І., ДАЙНЕКО П.М., СУДНІК-ВОЙЦИКОВСЬКА Б., ДЕМБІЧ І., ЗАХВАТОВИЧ М., ЗАХАРОВА М.Я. (2020). Конспект флори городищ Нижнього Придніпров'я. Чорноморськ. бот. ж., 16 (1): 6-39. doi: 10.32999/ksu1990-553X/2020-16-1-1

\footnotetext{
C) ${ }^{1}$ Moysiyenko I.I., ${ }^{1}$ Dayneko P.M., Sudnik-Wójcikowska B., Dembicz I., Zachwatowicz M., ${ }^{1}$ Zakharova M.Ya. ${ }^{1}$ Kherson State University, 27 Universytetska Str., Kherson, 73000, Ukraine

${ }^{2}$ Department of Plant Ecology and Environmental Conservation, Faculty of Biology, University of Warsaw, ul. Żwirki i Wigury 101, 02-089 Warsaw, Poland

${ }^{3}$ Faculty of Geography and Regional Studies Chair of Physical Geography, Department of Geoecology, University of Warsaw, ul. Krakowskie Przedmieście 30 00-927 Warsaw, Poland e-mail: ivan.moysiyenko@gmail.com Submitted 7 February $2020 \quad$ Recommended by D. Dubyna Published 18 April 2020
} 
Протягом минулих століть високий рівень сільськогосподарського виробництва призвів до значного зменшення степової рослинності. У трансформованих ландшафтах півдня України пам'ятки культури, такі як старі поселення, можуть бути потенціальними місцями для збереження біорізноманіття. Протягом останніх років (2010-2019) нами була приділена увага 18 городищам Нижнього Придніпров’я, площа яких варіюється від 1.1 до 18.7 га. Всі городища розташовуються на крутому березі Дніпра по обидві сторони, в основному між двома близько розташованими балками. Річкова тераса і балки були гарним природним захистом для городищ 3 трьох сторін, а з четвертої вони були захищені штучним ровом і валом. Окрім того, ключовою роллю розташування городищ був Дніпро як провідна водна магістраль для торгівлі та формування економічної структури у класичний та елліністичний періоди. Конспект флори городищ Нижнього Придніпров'я включає 526 видів судинних рослин, що відносяться до 279 родів, 74 родини, 3 класи та 2 відділи. На одному городищі росте від 125 до 290 видів судинних рослин, в середньому 178 . Городища 3 найбільшою площею характеризуються найбільшими показниками видів. Рівень созологічної цінності по окремим городищам неодноманітний. В цілому, на частину охоронюваних рослин припадає 30 видів (6\%). 3 них 10 видів включені до Червоної книги України (Astragalus borysthenicus, A. dasyanthus, Elytrigia stipifolia, Gymnospermium odessanum, Stipa capillata, S. lessingiana, S. pulcherrima, S. ucrainica, Tulipa biebersteiniana, T. gesneriana) та 20 видів до Червоного списку Херсонської області (Amygdalus nana, Bellevalia sarmatica, Bromopsis heterophylla, Dianthus andrzejowskianus, Ephedra distachya, Ferula capsica, Fraxinus excelsior, Hyacinthella leucophaea, Jurinea salicifolia, J. stochadifolia, Limonium platyphyllum, Linaria macroura, Linum czernjajevii, Prangos odontalgica, Quercus robur, Silene supina, Valeriana stolonifera, Veronica capsellicarpa, Vinca herbacea, Vitis sylvestris).

Ключові слова: судинні рослини, рідкісні угруповання, степ, охорона природи, Херсонська область

МОЙСИЕНКО И.И., ДАЙНЕКО П.М., СУДНИК-ВОЙЦИКОВСЬКАЯ Б., ДЕМБИЧ И., ЗАХВАТОВИЧ М., ЗАХАРОВА М.Я. (2020). Конспект флоры городищ Нижнего Приднепровья. Черноморськ. бот. ж., 16 (1): 6-39. doi: 10.32999/ksu1990$553 \mathrm{X} / 2020-16-1-1$

В течение прошлых веков высокий уровень сельскохозяйственного производства привел к значительному уменьшению степной растительности. В трансформированных ландшафтах юга Украины такие памятники культуры как городища могут быть потенциальными местами для сохранения степного биоразнообразия. В течение последних лет (2010-2019) мы исследовали 18 городищ Нижнего Приднепровья, площадь которых варьируется от 1.1 до 18.7 га. Все городища располагаются на крутом берегу Днепра по обе стороны, в основном между двумя близко расположенными балками. Речная терраса и балки были хорошей естественной защитой для городищ c трех сторон, a c четвертой стороны они были защищены искусственным рвом и валом. Кроме того, ключевой ролью расположения городищ был Днепр как ведущая водная магистраль для торговли и формирования экономической структуры в классический и эллинистический периоды. Конспект флоры городищ Нижнего Приднепровья включает 526 видов сосудистых растений, относящихся к 279 родам, 74 семьи, 3 класса и 2 отдела. На одном городище растет от 125 до 290 видов сосудистых растений, в среднем 178. Городища с наибольшей площадью характеризуются наибольшими показателями видов. Уровень созологичной ценности на отдельных городищах неоднообразна. В целом, на долю охраняемых растений приходится 30 видов (6\%). Из них 10 видов включены в Красную книгу Украины (Astragalus borysthenicus, A. dasyanthus, Elytrigia stipifolia, Gymnospermium odessanum, Stipa capillata, S. lessingiana, S. pulcherrima, S. ucrainica, Tulipa biebersteiniana, T. gesneriana) и 20 видов в Красный список Херсонськой области (Amygdalus nana, Bellevalia sarmatica, Bromopsis heterophylla, Dianthus andrzejowskianus, Ephedra distachya, Ferula capsica, Fraxinus excelsior, Hyacinthella leucophaea, Jurinea salicifolia, J. stochadifolia, Limonium platyphyllum, Linaria macroura, Linum czernjajevii, Prangos odontalgica, Quercus robur, Silene supina, Valeriana stolonifera, Veronica capsellicarpa, Vinca herbacea, Vitis sylvestris).

Ключевые слова: сосудистые растения, редкие сообщества, степь, охрана природы, Херсонская область 
The high potential of natural conditions and resources of the south of Ukraine affect the high level of agricultural production. Especially significant changes occurred in steppe zone in the south of Ukraine, where the area of steppe vegetation decreased forty-fold (steppe in the past covered circa $40 \%$ of the total territory of the country, while today steppe remnants survived only on $1 \%$ of this territory) [BURKOVSKYI et al., 2013]. Nowadays the Ukrainian steppe has been preserved only in several types of refugia: nature reserves, slopes of river valleys, loess ravines. Recent studies also demonstrated the high importance of habitat islands of anthropogenic origin as burial mounds or kurgans [DEÁK et al., 2016, 2018, 2020; MOYSIYENKO, SUDNIK-WÓJCIKOWSKA, 2006; SUDNIK-WÓJCIKOWSKA et al., 2011; VALKÓ et al., 2018], sacred groves and forests [BHAGWAT, RUTTE, 2006; BRANDT et al., 2013] cemeteries and graveyards [BARRETT, BARRETT, 2001]. Old settlements also have been the subject of different researches. Such earthwork values are represented in numerous publications concerning participation of thermophilous species in plant communities of earthworks [SUDER, 2011], role relicts of cultivation in vascular flora of settlements [CELKA, 2011]. Last years, researchers have been paying much attention to old settlements in Ukraine for the detection and conservation of steppe species [MOYSIYENKO at al., 2015, 2018, 2019; MOYSIYENKO, DAYNEKO, 2019; DAYNEKO, 2019].

Old settlements or «horodyshcha» in Slavic are ancient communities (or nowadays remnants of communities) where was created a characteristic defense complex (traditionally the settlements were surrounded by earthen ramparts with moats) [GAVRYLYUK, 2001; GAVRYlyUK, MATERA, 2016; LiSETSKII, PiCHURA, 2020]. Over time, ancient settlements have been left for many centuries, but in the interior of southern Ukraine they are an essential element to this days. Their value as a monument of archeology is fixed at the legislative level (all investigated settlements are objects of cultural heritage of national importance in Ukraine). However, in addition to the cultural and historical function of these sites, they perform an important conservation function by preserving a high level of restored quasinatural steppe vegetation. Identification and conservation of steppe sites is a very important direction of environmental researches in Ukraine.

The aim of this study was to summarize the data for the Lower Dnipro old settlements vascular flora and its specific peculiarities, as well as to assess the role of the settlements for the steppe flora preservation.

\section{Study area}

The characteristic features of the natural conditions and resources of the Lower Dnipro region are determined by its geographical location within the steppe zone of the Eastern European Plain. Loess is the most common geological surface formation in the region, reaching a thickness of several tens of meters. Under the loess on the whole territory along the Lower Dnipro presented Neogene deposits (limestone, sands, sandstones, marls and clays).

The climate of the Lower Dnipro as steppe zone is continental with mild winters and hot long summers. The sum of active temperature is $3200-3300^{\circ} \mathrm{C}$. The mean annual temperature is $9-10^{\circ} \mathrm{C}$. The average frost-free period duration is $170-180$ days. The region is characterized by a small amount of precipitation (350-420 $\mathrm{mm}$ per year) and intense solar radiation [MARYNYCH, SHYSHCHENKO, 2005].

Regarding geobotanical zoning, the Lower Dnipro region is located in three districts of the Black Sea and Azov steppe sub-province of the Pontic steppe province of the Steppe zone: Bug-Ingul district of grasses, grass meadows and vegetation of limestone outcrops, 


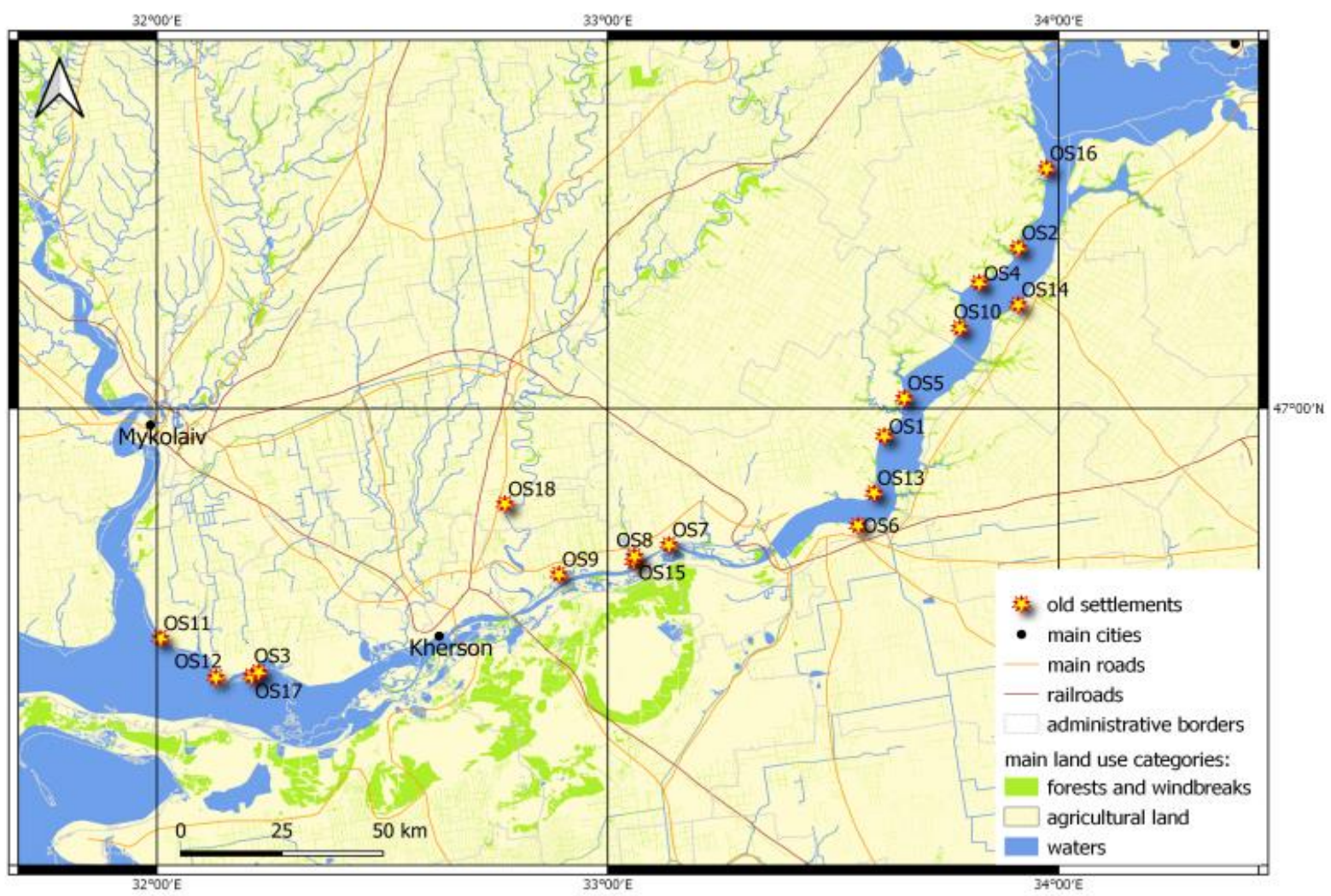

Fig. 1. Map of the location of the Lower Dnipro old settlements. Explanation: OS1 - Chervonomaiatske, OS2 - Gavrylivske, OS3 - Glyboka Prystan, OS4 - Hannivske, OS5 - Konsulivske, OS6 - Liubymivske, OS7 - Lvivske, OS8 - Male Tiagynske, OS9 - Poniativske, OS10 - Sablukivske, OS11 - Skelka, OS12 Stanislavske, OS13 - Staroshvedske, OS14 - Velykolepetykhske, OS15 - Velyke Tiagynske, OS16 Zolotabalkivske, OS17 - Zolotyi Mys, OS18 - Oleksandrivka-Roksanivka.

Lower Dnipro district of sand steppes, sands and reed beds, Dnipro-Azov district of grasses, wormwood-grass steppes and depression (pid - in Ukrainian) meadows [GEOBOTANICAL ZONING..., 1977]. In the administrative-territorial division, 17 old settlements are located in the Kherson region (Belozersky, Beryslavsky, Novovorontsovsky, Velikolepetetsky and Kakhovsky districts) and 1 - in the Mykolayiv region (Snihurivsky district (table 1).

All settlements located on the steep bank of the Dnipro River on both sides (Kherson and Mykolayiv Regions), mainly between two closely spaced ravines or «balkas» (fig. 1, 2). An exception is the settlement Oleksandrivka-Roksanovka, which is located on the right tributary of the Dnipro - the Ingulets River. The river terrace and ravines provided a natural protection for the settlements on three sides, and on the another side they were protected by artificial moat and shaft. The Dnipro was acknowledged as playing a key role in specific location of the earthworks as a leading waterway for trade and economic structure formation in the classical and Hellenistic periods. [GAVRYLYUK, MATERA, 2016].

The area of the settlements varies from 1.1 to 18.7 ha (table 1). The location specificity of the settlements on the terrace between the ravines causes a complex of characteristic adverse processes. Unfavorable geological processes at the sites of settlements are soil erosion and abrasion of the banks of the Dnipro estuary and the Kakhovka reservoir. A number of adversely anthropogenic factors, as excavation, driving, cattle grazing, fires effect on the of all settlements steppe vegetation. Beside afforestation (Chervonomaiatske, Gavrylivske, Konsulivske, Poniativske, Sablukivske, Zolotobalkivske), the limestone mining (Oleksandrivka-Roksanivka, Lvivske, Velykolepetykhske) is also common process. 

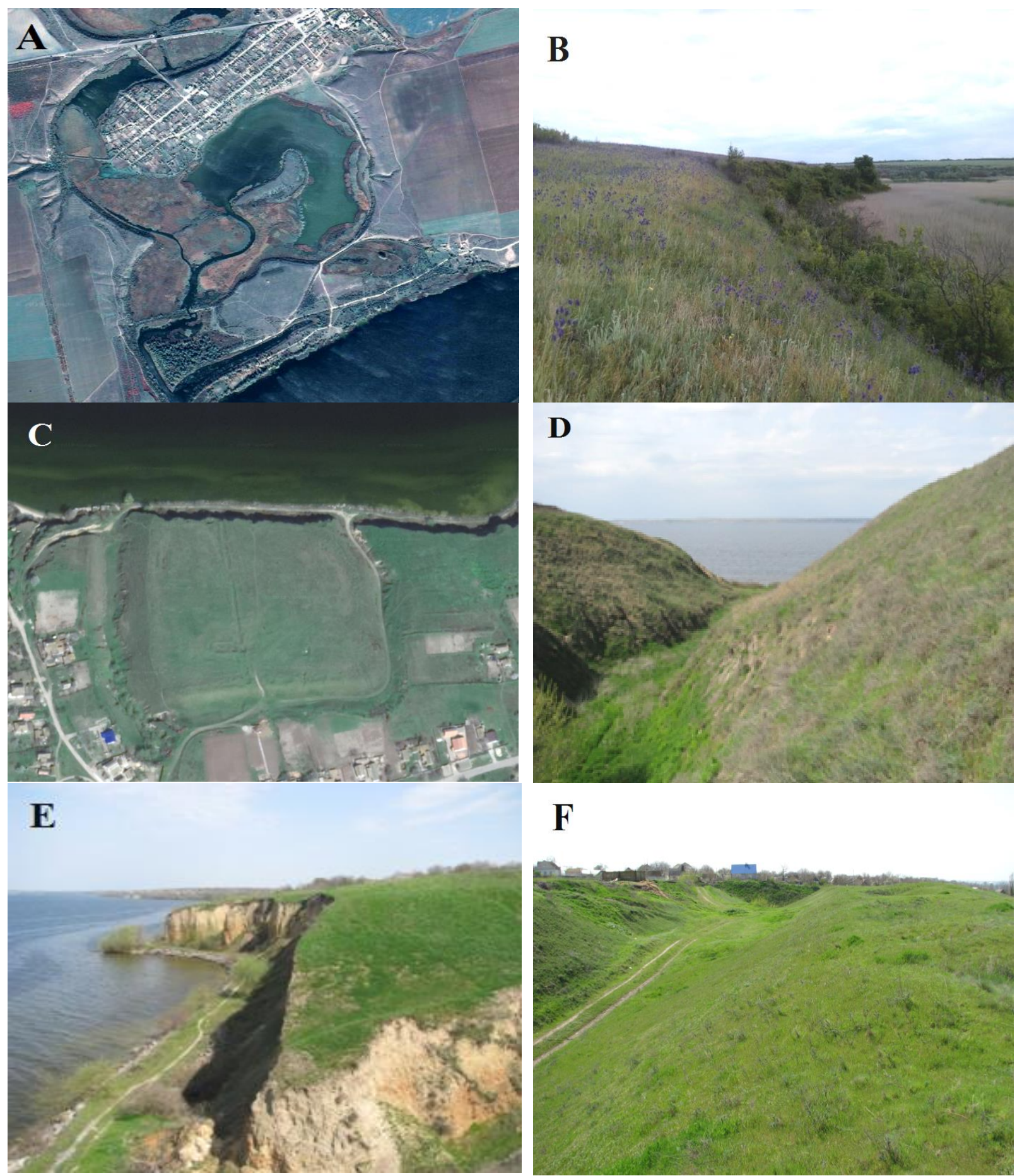

Fig. 2. The general view of some old settlements of the Lower Dnipro: A, B - Velyke Tiagynske old settlements; C, D, E, F - Liubymivske old settlement (A, C - view of the old settlements in the satellite image of the system Google Earth; B, D, E, F - photo by Moysiyenko I.I.).

Ancient settlements are diverse in accordance of their location relative to the present settlements. Given the proximity of the earthworks to the settlement can be divided into 3 groups: located directly in the middle of the existing settlements today, in their immediate vicinity, or significantly remote from them. 


\section{Material and methods}

During the last decade (2010-2019) the flora of the Lower Dnipro ancient settlements was investigated. The first results on 10 settlements have been presented before [MOYSIYENKO at al., 2018].

Table 1

General information of the investigated settlements of the Lower Dnipro

\begin{tabular}{|c|c|c|c|c|c|}
\hline № & Name & Coordinates & $\begin{array}{l}\text { Area, } \\
\text { hectare }\end{array}$ & Age & Nation \\
\hline \multicolumn{6}{|c|}{ Kherson region } \\
\hline 1. & Chervonomaiatske & $\begin{array}{l}46.572427 \\
33.364850\end{array}$ & 6.11 & III c. BC-IV c. AD & $\begin{array}{l}\text { Late Scythian settlement of } \\
\text { Sarmatian time }\end{array}$ \\
\hline 2. & Gavrylivske & $\begin{array}{l}47.250402 \\
33.911010\end{array}$ & 3.23 & IV-III c. BC & $\begin{array}{l}\text { settlement Late Bronze Late } \\
\text { Scythian settlement }\end{array}$ \\
\hline 3. & Glyboka Prystan & $\begin{array}{l}46.350405 \\
32.133205 \\
\end{array}$ & 2 & V c. BC-III c. AD & Ancient settlement \\
\hline 4. & Hannivske & $\begin{array}{l}47.114729 \\
33.492619\end{array}$ & 2.24 & II c. BC - II c. AD & Late Scythian settlement \\
\hline 5. & Konsulivske & $\begin{array}{l}46.572427 \\
33.364850\end{array}$ & 5.37 & I c. BC - II c. AD & $\begin{array}{l}\text { Late Scythian settlement of } \\
\text { Sarmatian time }\end{array}$ \\
\hline 6. & Liubymivske & $\begin{array}{l}46.485690 \\
33.331956\end{array}$ & 4.43 & $\begin{array}{l}\text { II c. BC - III c. AD, } \\
\text { VIII-X, XIV-XV c. } \\
\text { AD }\end{array}$ & Late Scythian settlement \\
\hline 7. & Lvivske & $\begin{array}{l}47.005812 \\
33.392745 \\
\end{array}$ & 9.24 & II c. BC - III c. AD & $\begin{array}{l}\text { Late Scythian settlement of } \\
\text { Sarmatian time }\end{array}$ \\
\hline 8. & Male Tiagynske & $\begin{array}{l}46.766520 \\
33.058668\end{array}$ & 6.3 & XIV-XVI c. AD & Iron Age, Scythian settlement \\
\hline 9. & Poniativske & $\begin{array}{l}46.738426 \\
32.891405 \\
\end{array}$ & 8.27 & II c. BC - II c. AD & Late Scythian settlement \\
\hline 10. & Sablukivske & $\begin{array}{l}47.074726 \\
33.470599\end{array}$ & 12 & I c. AD & Late Scythian settlement \\
\hline 11. & Skelka & $\begin{array}{l}46.381775 \\
32.002741\end{array}$ & 5.82 & $\begin{array}{l}\text { V-III c. BC., I c. } \\
\text { BC-III c. AD, VI- } \\
\text { V c. BC }\end{array}$ & Scythian settlement \\
\hline 12. & Stanislavske & $\begin{array}{l}46.343374 \\
32.074997\end{array}$ & 6.35 & $\begin{array}{l}\text { XII-XI c. BC, VI-V } \\
\text { c. BC, IV-II c. BC, } \\
\text { I c. BC - II c. AD, } \\
\text { III-IV c. AD }\end{array}$ & $\begin{array}{l}\text { settlement Late Bronze Age } \\
\text { Bilozerskoy culture; } \\
\text { settlement of Chernyakhiv culture }\end{array}$ \\
\hline 13. & Staroshvedske & $\begin{array}{l}46.520203 \\
33.352596 \\
\end{array}$ & 3.6 & II c. BC - III c. AD & $\begin{array}{l}\text { Late Scythian settlement of } \\
\text { Sarmatian time }\end{array}$ \\
\hline 14. & Velykolepetykhske & $\begin{array}{l}47.162411 \\
33.910218 \\
\end{array}$ & 4.82 & No data & Late Scythian settlement \\
\hline 15. & Velyke Tiagynske & $\begin{array}{l}46.453669 \\
33.032777 \\
\end{array}$ & 18.7 & I-XVI c. AD & $\begin{array}{l}\text { Late Scythian settlement, } \\
\text { Golden Horde period }\end{array}$ \\
\hline 16. & Zolotobalkivske & $\begin{array}{l}47.223185 \\
33.582336\end{array}$ & 1.1 & II c. BC - III c. AD & Late Scythian settlement \\
\hline 17. & Zolotyi Mys & $\begin{array}{l}46.344082 \\
32.123604 \\
\end{array}$ & 1.3 & III c. BC - III c. AD & Ancient settlement \\
\hline \multicolumn{6}{|c|}{ Mykolayiv region } \\
\hline 18. & $\begin{array}{l}\text { Oleksandrivka- } \\
\text { Roksanivka }\end{array}$ & $\begin{array}{l}46.510224 \\
32.462166\end{array}$ & 2.83 & V-IV c. BC & settlement of Chernyakhiv culture \\
\hline
\end{tabular}

In this article we present the results of the research of all 18 ancient settlements of the Lower Dnipro (the section of the Dnipro is about $220 \mathrm{~km}$ long in Kherson region and $40 \mathrm{~km}$ of Ingulets as right tributary of the Dnipro river in Kherson and Mykolaiv regions).

The study of each site was conducted at least 3 times according to the season: spring, summer and autumn. The data were compiled in Table A (Appendix 1) which contained the following additional information about each taxon: its occurrence and abundance (estimated according to a 3-point scale: 1 - sporadic, 2 - infrequent, 3 - common), functional group (hs habitat specialist, $\mathrm{g}$ - generalist), species life form, species life span, it's status in the 
historical-geographical classification [KORNAś, 1968, 1977], the number of old settlements on which the species occurs, and origin in the case of alien species. Alien species were identified on the basis of publication by [PROTOPOPOVA, 1991].

Floristic analyses was conducted in which such selected elements as species richness and spectra of selected species groups were assessed, i.e. life forms and synanthropic groups. The specificity of the earthworks flora was determined. The following scale was used to assess the frequency category of the species: I - rare (< 17\% i.e. 1-3 earthworks), II relatively rare (18-34\% i.e. 4-6 earthworks), III - not rare (35-50\% i.e. 7-9 earthworks), IV relatively frequent (51-67\% i.e. 10-12 earthworks), $\mathrm{V}$ - frequent (68-84\% i.e. 13-15 earthworks), VI - common (85-100\% i.e. 16-18 earthworks).

To check what is the level of synanthropization of the flora of old settlements we used geographical-historical classification by [KORNAŚ, 1968, 1977], and assigned all species to the following groups as indegenious species (non-synanthropic species, hemiapophytes, euapophytes and ekiophytes) and anthropophytes (archaeophytes and kenophytes).

To assess plant adaptation to certain ecological conditions we used classification spectrum of life forms wich includes certain groups as hemicryptophytes, therophytes, geophytes, phanerophytes, chamaephytes, hemicryptophytes-chamaephytes.

The identification of vascular plant species was held in the Kherson State University Laboratory of Plant Ecology and Environmental Protection. Herbarium specimens are stored in the collection of Kherson State University (KHER). Names of plant species are given in Latin according to [MOSYAKIN \& FEDORONCHUK, 1999]. We have used QGIS 3.10.1, Open Street Map, Natural Earth and Google Earth Pro to prepare mapping and spatial analysis of the Lower Dnipro region.

\section{Results}

1. Biodiversity of the old settlements flora

A list of the flora of vascular plants includes 526 species from 18 old settlements of the Lower Dnipro. Total species richness ranged from 125 to 290 (178 species per archeological site in average) (fig. 3, Appendix 1). The species belong to 279 genera, 74 families, 3 classes and 2 divisions. The vast majority of flora species belong to the Magnoliophyta division (99.8\%). The Pinophyta division (0.2\%) is represented by one family Ephedraceae and one species - Ephedra distachya. The following families were represented by the greatest number of taxa (fig. 4): Asteraceae (73 species), Poaceae (49), Fabaceae (39), Lamiaceae (33), Brassicaceae (29), Caryophyllaceae (26), Rosaceae (24), Boraginaceae (20), Scrophulariaceae (17), Chenopodiaceae (16), Apiaceae (13) (Fig. 3). Genera represented by the highest number of taxa were, as follows: Veronica (13), Astragalus (9), Artemisia (8), Chenopodium (7), Euphorbia (8), Galium (8).

About 242 species (46.0\% of the total ancient settlements flora) of the first (I) frequency class (fig. 5) were considered as rare (on 1-3 earthworks only). The group of common species recorded on 16-18 of the studied hillforts (frequency class VI) made up about $8 \%$ of the flora: Agropyron pectinatum (18), Anisantha tectorum (18), Arenaria serpyllifolia (18), Artemisia austriaca (18), Bromus squarrosus (18), Buglossoides arvensis (18), Convolvulus arvensis (18), Elytrigia repens (18), Eryngium campestre (18), Falcaria vulgaris (18), Festuca valesiaca (18), Galium humifusum (18), Kochia prostrata (18), Medicago falcata (18), Poa angustifolia (18), Poa bulbosa (18), Pterotheca sancta (18), Securigera varia (18), Senecio vernalis (18), Sisymbrium loeselii (18), Tragopogon major (18), Verbascum phoeniceum (18), Alyssum desertorum (17), Anthemis ruthenica (17), Artemisia lerchiana (17), Centaurea diffusa (17), Chondrilla juncea (17), Galium aparine (17), Geranium pusillum (17), Koeleria cristata (17), Lactuca serriola (17), Marrubium praecox (17), Onopordum acanthium (17), Achillea setacea (16), Capsella bursa-pastoris (16), Descurainia sophia (16), Galium spurium (16), Potentilla recta (16), Rosa canina (16), Teucrium polium (16), Thymus dimorphus (16). 


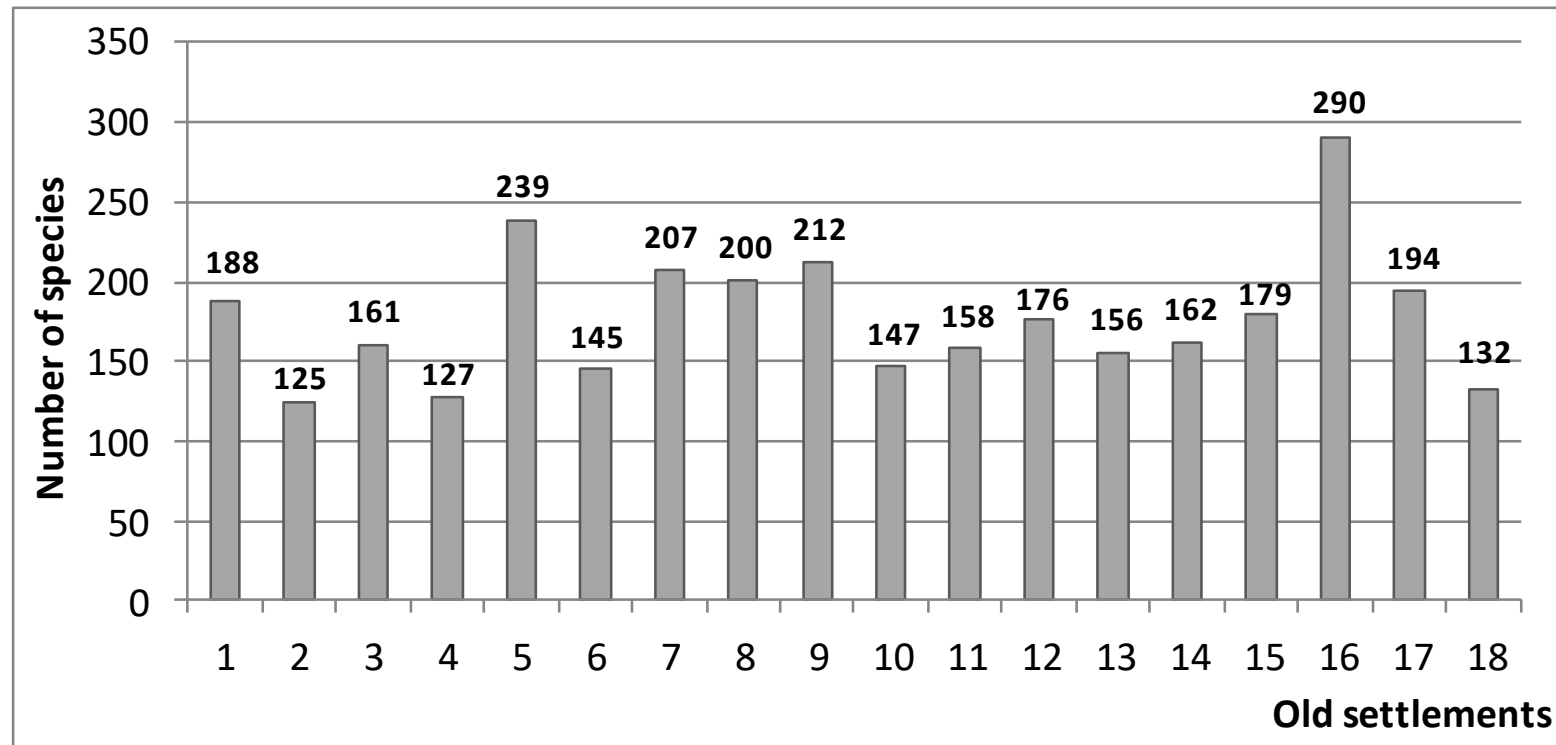

Fig. 3. Number of species in the compared flora of the Lower Dnipro old settlements (the total number of species in each old settlements is indicated at the top of the bar). Explanation: 1 - Chervonomaiatske, 2 Gavrylivske, 3 - Glyboka Prystan, 4 - Hannivske, 5 - Konsulivske, 6 - Liubymivske, 7 - Lvivske, 8 - Male Tiagynske, 9 - Oleksandrivka-Roksanivka, 10 - Poniativske, 11 - Sablukivske, 12 - Skelka, 13 Stanislavske, 14 - Staroshvedske, 15 - Velykolepetykhske, 16 - Velyke Tiagynske, 17 - Zolotabalkivske, 18 - Zolotyi Mys.

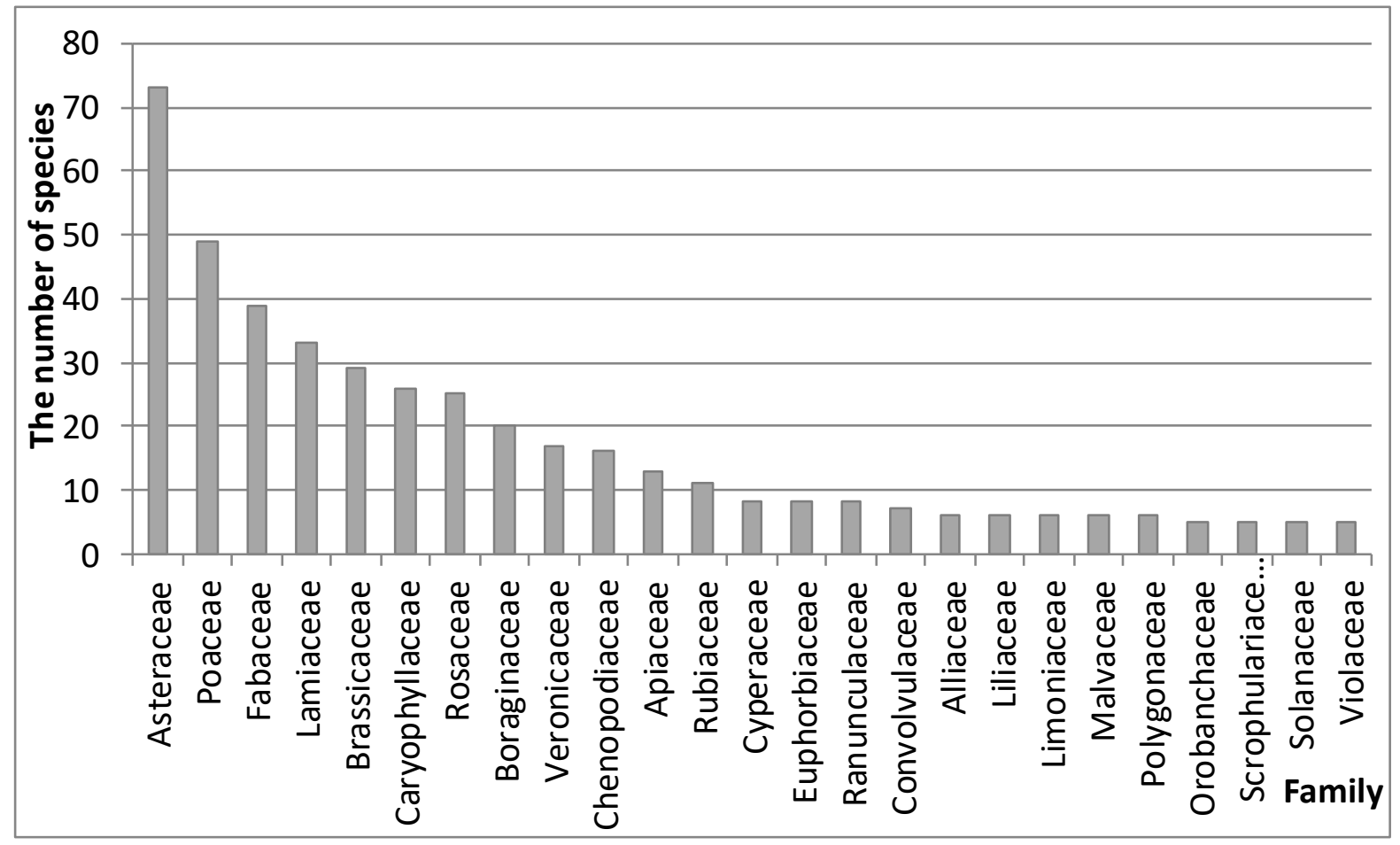

Fig. 4. The most important families (in terms of species number) in the total flora of the Lower Dnipro old settlements. 


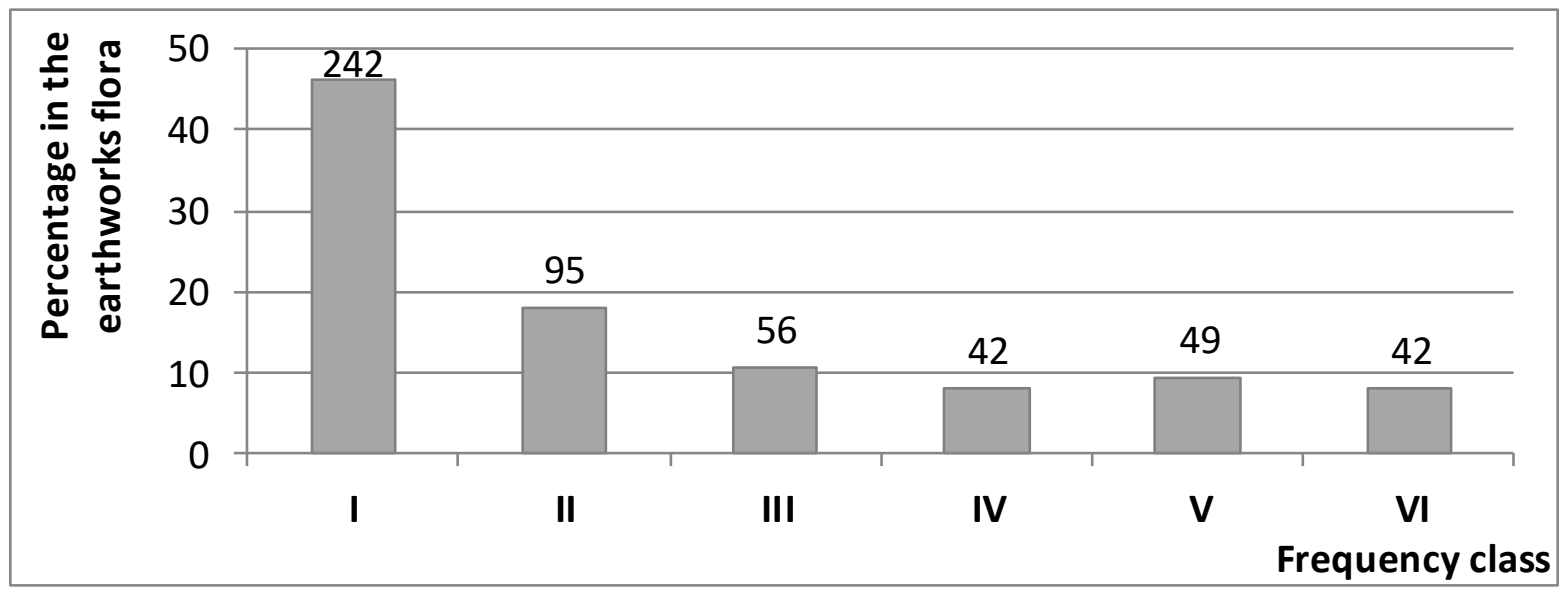

Fig. 5. Subdivision of the flora of the Lower Dnipro old settlements by frequency class (the total number of species in each category is indicated at the top of the bar). Explanation: I - rare (< 17\% i.e. 1-3 earthworks), II - relatively rare (18-34\% i.e. 4-6 earthworks), III - not rare (35-50\% i.e. 7-9 earthworks), IV - relatively frequent (51-67\% i.e. 10-12 earthworks), V - frequent (68-84\% i.e. 13-15 earthworks), VI - common (85-100\% i.e. 16-18 earthworks).

\section{Spectrum of life forms}

The spectrum of life forms in the flora of ancient settlements corresponds basically to that of the flora of the Pontic grass steppe zone [SUDNIK-WÓJCIKOWSKA, MoYSIYENKO, 2006]. Hemicryptophytes (46.1\%) are the dominating group of species (fig. 6). The second largest group were therophytes (32.5\%). They are less numerous compared to hemicryptophytes and occupy smaller areas.

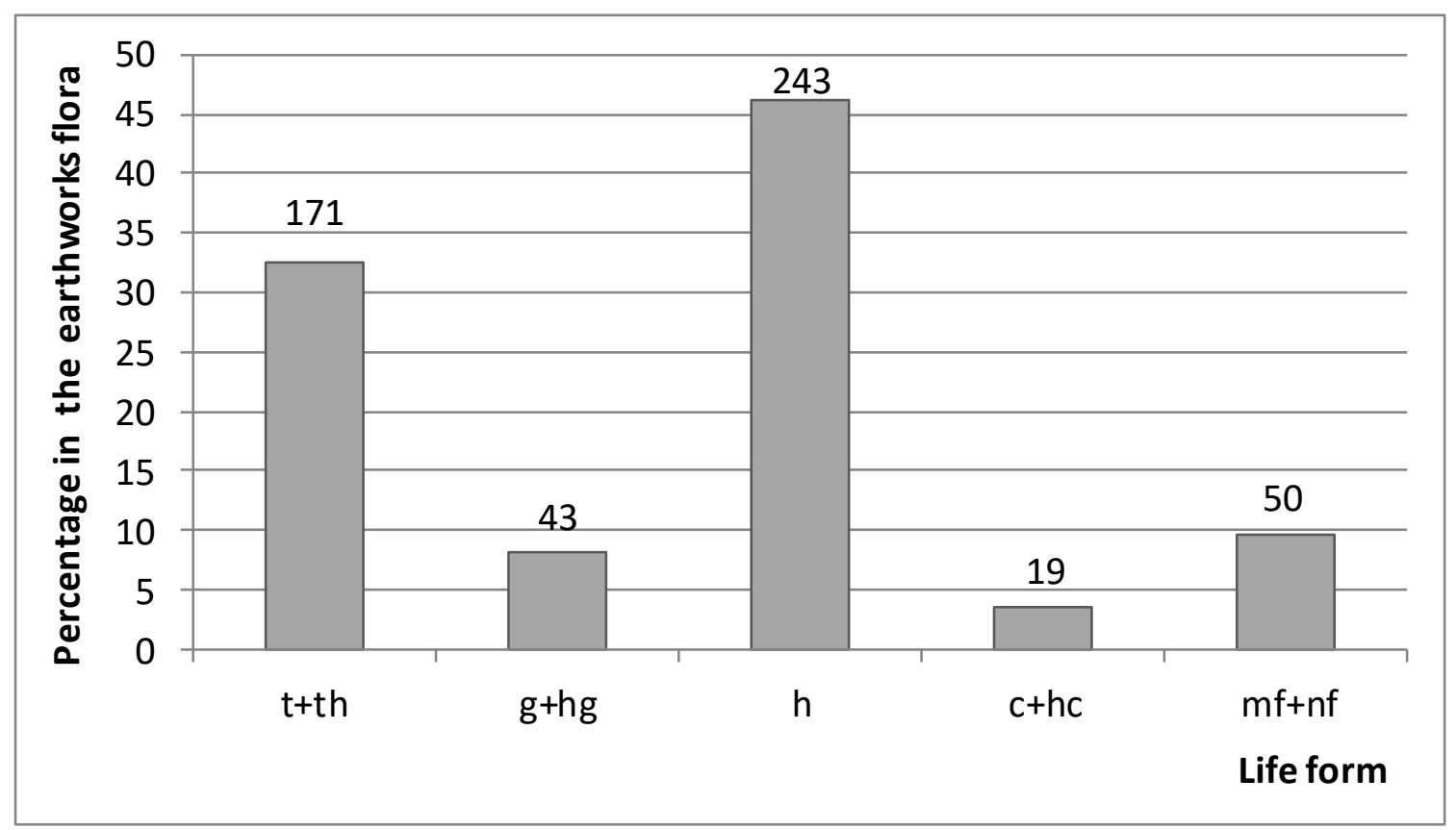

Fig. 6. Spectrum of life forms in the flora of the Lower Dnipro old settlements (for abbreviations see Appendix 1). The absolute number of species in each category is indicated at the top of the bar.

In the studied areas phanerophytes comprised $9.7 \%$ of the flora of ancient settlements. In the Lower Dnipro zone the following most frequent species of megaphanerophytes were recorded: Acer negundo, A. platanoides, A. tataricum, Ailanthus altissima, Armeniaca vulgaris, Cerasus vulgaris, Elaeagnus angustifolia, Gleditsia triacanthos, Fraxinus pennsylvanica, Pinus palassiana, Prunus cerasifera, $P$. diversifolia, P. stepposa, Robinia 
pseudoacacia, Quercus robur, Ulmus campestris, U. laevis, U. pumila. Nanophanerophytes Amygdalus nana, Amorpha fruticosa, Berberis vulgaris, Cornus sanguinea, Cotinus coggygria, Crataegus monogyna.

The other life forms groups played a lesser represented in the flora the earthworks in the Lower Dnipro zone: chamaephytes comprised 16 species or 3\% of the old settlements flora, and hemicryptophytes-chamaephytes -3 species or $0.6 \%$. The following species of native chamaephytes were recorded: Artemisia absinthium, A. austriaca, A. marschalliana, A. santonica, A. taurica, Astragalus albidus, Kochia prostrata, Minuartia setacea, Nepeta cataria, Solanum dulcamara, Teucrium chamaedrys, Thalictrum minus, Thymus dimorphus.

3. Spectrum of species groups in the historical-geographical classification of plants

The majority of species of the earthwork flora of the Lower Dnipro zone are native plants (398, i.e. about $75.5 \%$ of flora). More than a half of this group (236) and $45 \%$ of the total number of species (fig. 7), are the plants not entering the habitats altered by man, i.e. non-synanthropic, represented on the ancient settlements mostly by steppe plants and halophytes. Non-synanthropes occurring with the highest frequency: Festuca valesiaca, Kochia prostrata, Koeleria cristata, Rosa canina, Teucrium polium, Thymus dimorphus, Verbascum phoeniceum.

Depending on the level of transformation of the habitats into which they penetrate, apophytes can be subdivided into 3 groups: hemiapophytes (18.6\% of the total flora), euapophytes (10.6\%) and ekiophytes (1.3\%) (fig. 7). With respect to frequency, hemiapophytes distinctly predominated over euapophytes; 16 hemiapophytes belonged to frequency class VI (Achillea setacea, Agropyron pectinatum, Alyssum desertorum, Arenaria serpyllifolia, Artemisia austriaca, Artemisia lerchiana, Chondrilla juncea, Eryngium campestre, Falcaria vulgaris, Galium humifusum, Marrubium praecox, Medicago falcata, Poa angustifolia, Poa bulbosa, Pterotheca sancta, Securigera varia; but only 6 euapophytes represented the above frequency class (Anthemis ruthenica, Convolvulus arvensis, Elytrigia repens, Galium aparine, Senecio vernalis, Tragopogon major).

On all the ancient settlements, to a smaller or greater extent, alien species were found i.e. anthropophytes (fig. 7). They comprised $24.5 \%$ of the flora of earthworks in the Lower Dnipro zone and represented mainly families such as: Asteraceae (15 species),

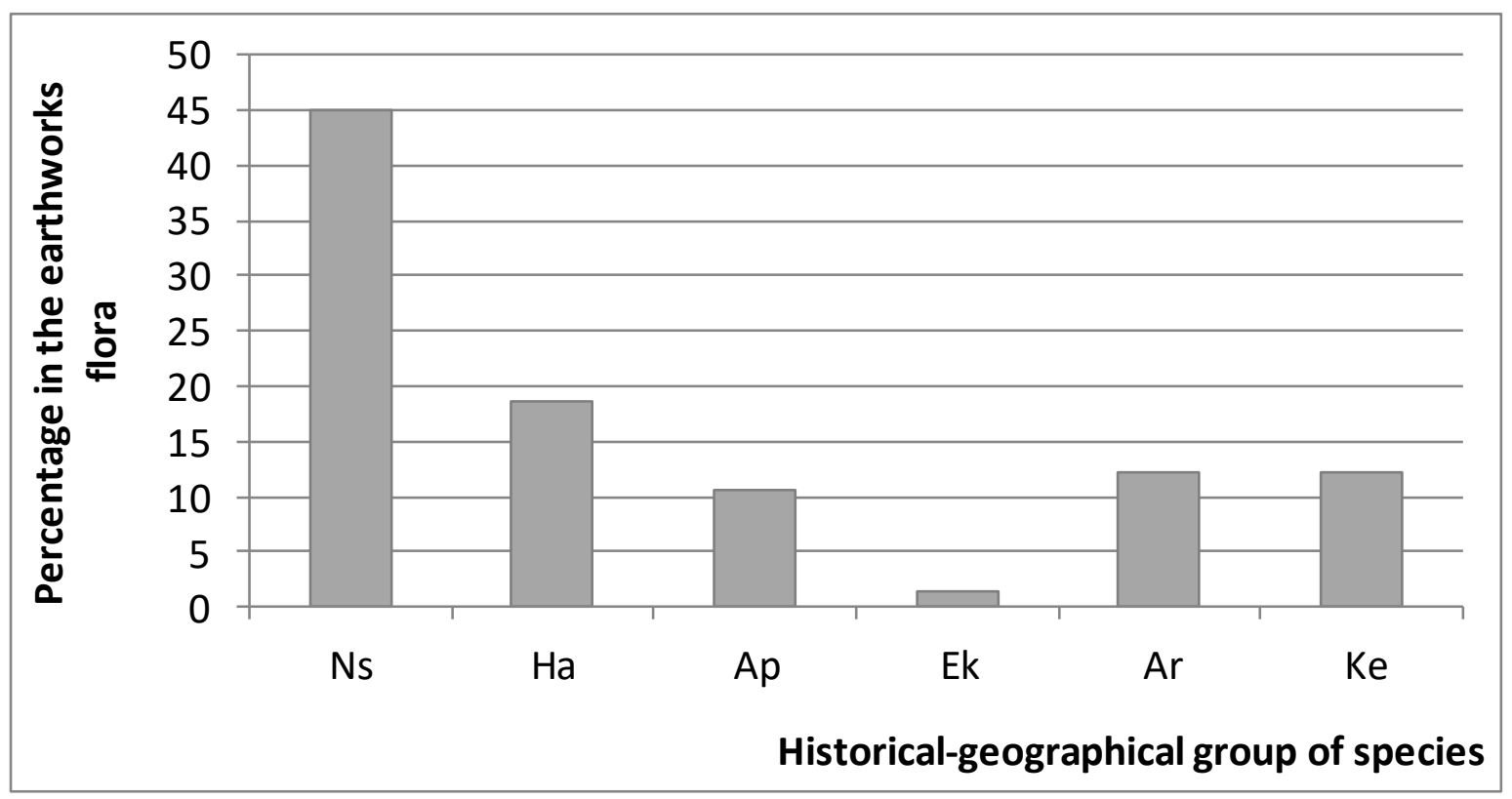

Fig. 7. Historical-geographical classification of the total flora of the Lower Dnipro old settlements. For abbreviations see Appendix 1. 
Brassicaceae (13), Poaceae (12), Fabaceae (11), Chenopodiaceae (9) Boraginaceae (7), Rosaceae (6), Lamiaceae (4), Malvaceae (4), Solanaceae (4); other families are represented by 1-3 species each.

Among alien species archaeophytes and kenophytes make up almost the same percentage, characteristic dominance is no evidence. Archaeophytes represented 64 species (12.2\% of the total flora of old settlements). The most frequently occurring archaeophytes (frequency class VI) were: Anisantha tectorum, Ballota nigra, Bromus squarrosus, Buglossoides arvensis, Capsella bursa-pastoris, Descurainia sophia, Galium spurium, Geranium pusillum, Lactuca serriola, Onopordum acanthium, Sisymbrium loeselii.

Total number of kenophytes is 65 species or $12.3 \%$ of the total flora of the old settlements. Only 1 species of kenophytes represented frequency class VI (Centaurea diffusa) and 3 species frequency class V: Cardaria draba, Elaeagnus angustifolia, Rumex patientia. Another kenophytes were included to first frequency classes.

An appreciable number of native plants indicate a high level of preservation of the objects (fig. 8.). The contribution of native species in old settlements varied much from $62.1 \%$ (Liubymivske) to $81.1 \%$ (Velyke Tiagynske). The contribution of native species to the alien species is $3: 1$.

\section{Discussion}

Despite of relatively small size of old settlements, they are characterized by a high level of vascular plant richenss. The flora of the ancient settlements represent $10.3 \%$ of the flora of Ukraine, which includes 5.100 species [MOSYAKIN, FEDORONCHUK, 1999] and 26.0\% of the flora of the Northern Black Sea, which includes 2.025 species [MOYSIYENKO, 2013]. The total number of vascular plant species of the old settlements was higher than the total number of species in the Ascania-Nova Biosphere Reserve (515 species according to [SHAPOVAL, 2012]). The 75.5\% (398 species) of all species that occurred on the earthworks were native plants. More than a half of this group (i.e. 237 species) and $45 \%$ of the total number of species were the non-synanthropic plants. An impressivenumber of indigenous and non-synanthropic plants demonstrated a high level of conservation of the native flora, especially steppe one.

The high number of therophytes in the flora of the Lower Dnipro old settlements is due to the less favourable geo-physical conditions in the south of Ukraine (lower annual rainfall, higher insolation and soil salinity level) and anthropogenic influences. On the earthworks, in places disturbed by animals and man, ecological niches are formed which are filled first by therophytes. Most of them (81 from 170), are alien species e.g. many weeds from the surrounding fields. It indicates that the flora of the settlements is, to some extent, the subject of anthropogenic transformation.

As previously mentioned, the species richness of the settlements flora is negatively affected by a number of anthropogenic factors: excavation (official and non-official), afforestation, limestone extraction, clogging, grazing, fires, etc.

The process of overgrowing of old settlements as well as kurgans with trees and shrubs poses a serious threat for biodiversity across the steppe zone. Shrubs and groups of trees displace light-loving steppe species. In studied area we noted earthworks partly overgrown with forest, e.g. within Poniativka (Bilozerka district), Konsulivka (Berislav district) and others. It is important to emphasize that woody encroachment is also a seriuos problem from the archaeological point of view (trees can demolish the stucture of the ancient buildings or decreases the landscape value of the sites (ancient forms are not visible)). 


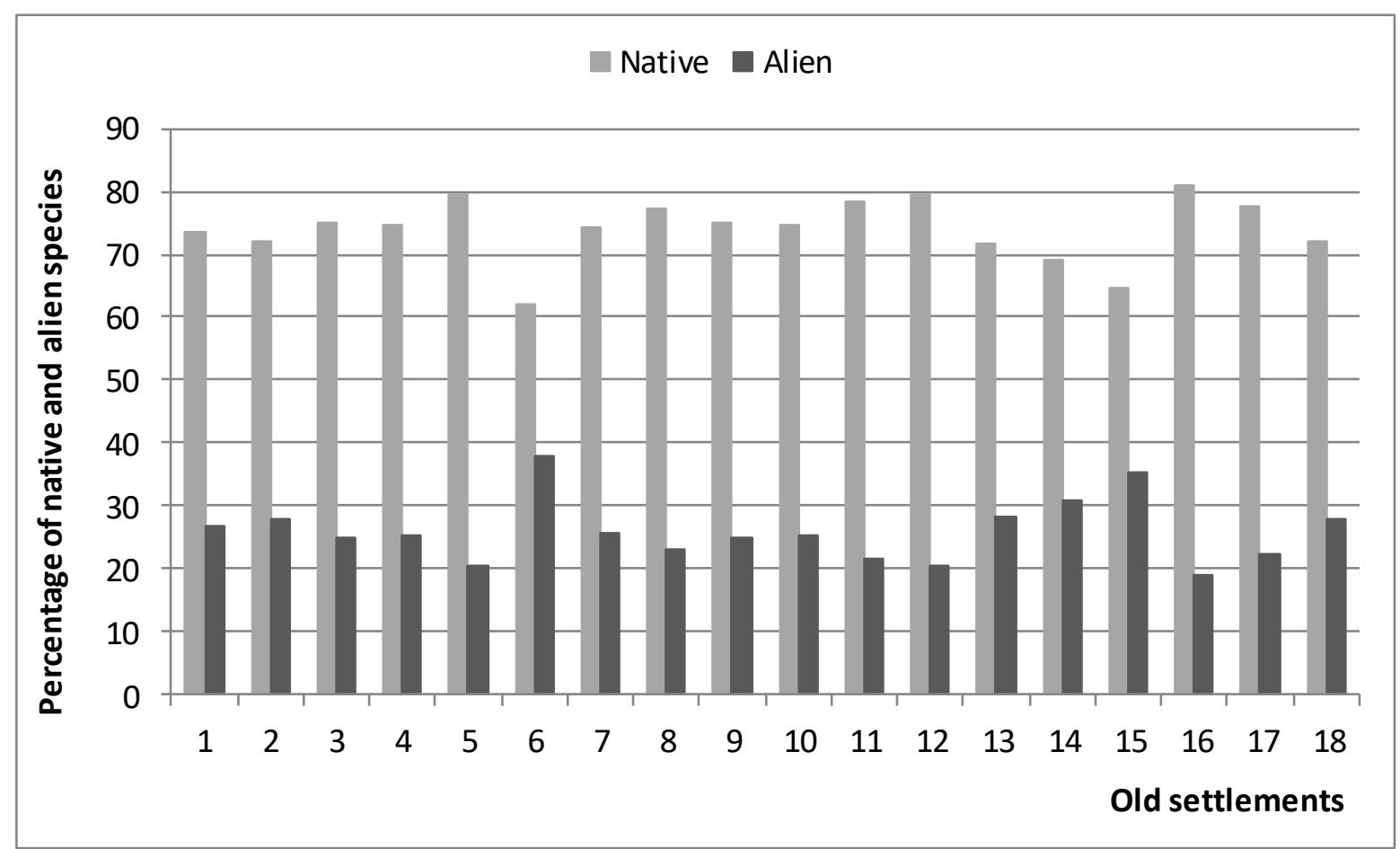

Fig 8. Contributions of native and alien species to the compared flora of old settlements. Explanation: 1 Chervonomaiatske, 2 - Gavrylivske, 3 - Glyboka Prystan, 4 - Hannivske, 5 - Konsulivske, 6 Liubymivske, 7 - Lvivske, 8 - Male Tiagynske, 9 - Oleksandrivka-Roksanivka, 10 - Poniativske, 11 Sablukivske, 12 - Skelka, 13 - Stanislavske, 14 - Staroshvedske, 15 - Velykolepetykhske, 16 - Velyke Tiagynske, 17 - Zolotabalkivske, 18 - Zolotyi Mys.

Simultaneously, the studied cultural monuments have a great conservation value. In the study area, we identified 30 species of protected vascular plants, of which 10 plant species are included in the Red Data Book of Ukraine [RED DATA BooK OF UKRAINE, 2009]: Astragalus borysthenicus, A. dasyanthus, Elytrigia stipifolia, Gymnospermium odessanum, Stipa capillata, S. lessingiana, S. pulcherrima, S. ucrainica, Tulipa biebersteiniana, T. gesneriana; 20 plant species are included in the Red List of Kherson Province: Amygdalus nana, Bellevalia sarmatica, Bromopsis heterophylla, Dianthus andrzejowskianus, Ephedra distachya, Ferula caspica, Fraxinus excelsior, Hyacinthella leucophaea, Jurinea salicifolia, J. stochadifolia, Limonium platyphyllum, Linaria macroura, Linum czernjajevii, Prangos odontalgica, Quercus robur, Silene supina, Valeriana stolonifera, Veronica capsellicarpa, Vinca herbacea, Vitis sylvestris [CHERVONYI SPYSOK KHERSONSKOI OBLASTI, 2013].

Our previous research also concerned ancient objects such as kurgans (mostly Scythian and Sarmatian burial mounds). In the same zone of the grass steppe, we tested 26 mounds with a total area of about 20 ha [SUDNIK-WÓJCIKOWSKA, MOYSIYENKO, 2006]. On a much smaller area, we have found 18 protected species, recorded in the Red Book of Ukraine or Red List of Kherson Province.

Our results emphasize floristic specificity of historical structures as old settlements in agriculturally transformed steppe zone. They could play an important role in steppe phytodiversity conservation, and give perspective for the future steppe restoration actions. Therefore, they should be subject to legal protection, and only moderate cattle grazing may be allowed.

\section{Acknowledgments}

We would like to express our gratitude to Serhii Niemtsev, Denys Sikoza and Andriy Lopushynskyi for inestimable help with data concerning location, age and area of old settlements and comments from archeologists point of view. We also thank Nadia Scobel, Anna Mala and Olena Raksha for the company during expeditions. This research is supported by the Swedish Science Council (Vetenskapsrådet) project N 2012-06112. 


\section{References}

Barrett G.W., BARretT T.L. (2001). Cemeteries as repositories of natural and cultural diversity. Conserv. Biol., 15(6): 1820-1824.

Bhagwat S.A., RuTTE C. (2006). Sacred groves: potential for biodiversity management. Front. Ecol. Environ., 4(10): 519-524.

Brandt J.S., Wood E.M., Pidgeon A.M., Han L.X., Fang Z., Radeloff V.C. (2013). Sacred forests are keystone structures for forest bird conservation in southwest China's Himalayan Mountains. Biol. Conserv., 16: 34-42.

Burkovskyi O.P., VASYliuk O.V., YenA A.V., KuZEMKo A.A., Movchan Y.I., MoysiYenKo I.I., SIRENKO I.P. (2013). Ostanni stepy Ukrayiny: buty chy ne buty? Kyiv: Geoprint, 38 p. (in Ukrainian)

CELKA Z. (2011). Relics of cultivation in the vascular flora of medieval West Slavic settlements and castles. Biodiv. Res. Conserv., 22: 1-110.

CHERVONYI SPYSOK KHERSONSKOI OBLASTI (2013). Rishenia XXVI sesii Khersonskoi oblasnoi rady VI sklykannia № 893 vid 13.11.2013. Kherson: 13 p. (in Ukrainian)

DAYNEKO P.M. (2019). Species richness of vascular plants on the ancient settlements of the Lower Dnieper. Biology: from a molecule up to the biosphere. Materials of the XIV International Young Scientists Conference, Kharkiv, 27-29 November, 2019: 147-149.

DeÁK B., Tóthmérész B., VAlKó O., Sudnik-WojCiKowska B., Moysiyenko I.I., Bragina T.M., Apostolova I., Dembicz I., BYKov N.I., TÖRÖK P. (2016). Cultural monuments and nature conservation: a review of the role of kurgans in the conservation and restoration of steppe vegetation. Biodivers. Conserv., 25(3): 1-18.

DeÁK B., TölgYesi C., Kelemen A., BÁtori Z., Gallé R., Bragina T.M., Yerkin A.I., VAlKó O. (2018). The effects of micro-habitats and grazing intensity on the vegetation of burial mounds in the Kazakh steppes. Plant Ecol. Divers., 10: 509-520.

DeÁk B., VAlKó O., NAgy D.D., TÖRÖK P., Torma A., LöRinczi G., Kelemen A., Nagy A., Bede Á., MizSER S.Z., CSATHÓ A.I., TÓTHMÉRÉSZ B. (2020). Habitat islands outside nature reserves - threatened biodiversity hotspots of grassland specialist plant and arthropod species. Biological Conservation, 241: 108-254.

GAVRYLYUK N.A. (2001). Slave trade and wine trade as the basic branches of Olbia-Barbaric Export-Import in Nothern Black Sea Area (VI-III cc. BC). Olviia ta antychnyi svit. Materialy naukovykh chytan prysviachenykh 75-richchiu utvorennia istoryko-arkheolohichnoho zapovidnyka «Olviia», Kyiv, 1922 November, 2001: 151-152. (in Ukrainian)

GAVRYlyUK N.A., MATERA M. (2016). «Late Scythian» or «Post-Scythian» sites of the lower Dnipro region. Archeology, 14: 121-135. (in Ukrainian)

Geobotanical zoning of the Ukraninan SSR (1977). Lavrenko E.M. (ed). Kyiv: Academy of Sciences of the USSR, 306 p. (in Ukrainian)

KORNAŚ J. (1968). A geographical-historical classification of synantropic plants. Mater. Zakl. Fitosoc. Stos. $U W ., 25:$ 33-41.

KORNAŚ J. (1977). Analiza flor synantropijnych. Wiad. Bot., 21: 85-91.

LISETSKII F.N., PICHURA V.I. (2020). Catena linking of landscape-geochemical processes and reconstruction of pedosedimentogenesis: a case study of defensive constructions of the mid-17th century, South Russia. Catena, 187: 104-300.

MARYNYCH O.M., SHYSHCHENKO P.G. (2005). Fizychna Heohrafiia Ukrainy. Kyiv, 511p. (in Ukrainian)

MOYSIYENKO I.I., DAYNEKO P.M. (2019). The role of the Old-Swedish hillfort in phytodiversity conservation. Advances in Botany and Ecology. Materials of the International Conference of Young Scientists, Kharkiv, 06-09 September, 2019: 38.

MOYSIYENKO I.I. (2013). Floristic diversity and taxonomic structure of the flora of the Pivnichne Prychornomoria (Northern Black Sea Coastal Plain). Chornomors'k. bot. z., 9(1): 41-56. (In Ukrainian)

Moysiyenko I.I., DAYneko P.M., Zachwatowicz M., Dembicz I., SudNiK-WóJCikowska B. (2019). An annotated list of the flora of the projected botanical reserve «Staroshvedsky» (Kherson region, Ukraine). Chornomors' $k$. bot. z., 15(2): 185-201. (In Ukrainian)

Moysiyenko I.I., Dembicz I., SUDNIK-WóJCIKOWSKA B., ZAChWATOWICZ M., Kuns B., ZaKharova M. (2018). Ancient settlements as refuges for steppe flora in southern Ukraine. Vegetation survey 90 years after the publication of Braun-Blanquet's textbook - new challenges and concepts Materials of the 27th Congress of the European Vegetation Survey, Wroctaw (Poland), 23-26 May, 2018: 13.

MOYSIYENKO I.I., SUDNIK-WÓJCIKOWSKA B. (2006). The flora of kurgans in the desert Steppe zone of southern Ukraine. Chornomors' 'k. bot. z., 2(1): 5-35. 
MOYSIYENKo I.I., SUDNIK-WÓJCIKOWSKA B., DEMBICZ I., SHAPOSHNIKOVA A. (2015). Preservation of phytodiversity on the kurgans. Scriptorium nostrum, 1-2: 261-280.

Mosyakin S.L., FedORONCHUK M.M. (1999) Vascular plants of Ukraine. A nomenclatural checklist. Kyiv: National Academy of Sciences of Ukraine, 346 p.

Protopopova V.V. (1991) Sinantropnaya flora Ukrainyi i puti ee razvitiya. Kyiv: Naukova dumka, 204 p. (in Ukrainian)

RED Data Book of Ukraine. Vegetable Kingdom (2009). Didukh Ya.P. (ed). Kyiv: Globalconsaltyng, 912 p. (in Ukrainian)

SHAPOVAL V.V. (2012). Flora sudynnyh roslyn Askanijs 'kogo stepu [Flora of vascular plants of Askania steppe]. FOP Andrjejev O. V., Askanija Nova. (in Ukrainian)

SUDER D. (2011). Participation of thermophilous species in plant communities of earthworks and castle ruins in theWestern Carpathians. Ann. UMCS, Biol., 66(2): 21-31.

SUDNIK-WÓJCIKOWSKA B., MoYsIYENKO I.I. (2006). The flora of kurgans in the West Pontic Grass steppe zone of Southern Ukraine. Chornomors'k. bot. z., 2(2): 14-44.

SUDNIK-WÓJCIKOWSKA B., MOYSIYENKO I.I., ZACHWATOWICZ M., JABŁOŃSKA E. (2011). The value and need for protection of kurgan flora in the anthropogenic landscape of steppe zone in Ukraine. Plant Biosystems, 145(3): 638-653.

VAlkó O., Tóth K., Kelemen A., Miglécz T., Radócz S., Sonkoly J., Tóthmérész B., TöröK P., DeAK B. (2018). Cultural heritage and biodiversity conservation-plant introduction and practical restoration on ancient burial mounds. Nat. Conserv., 24: 65. 
Moysiyenko I.I., Dayneko P.M., Sudnik-Wójcikowska B., Dembicz I., Zachwatowicz M., Zakharova M.Ya.

\begin{tabular}{|c|c|c|c|c|c|c|c|c|c|c|c|c|c|c|c|c|c|c|c|c|c|}
\hline urds ə઼!า & 29 & 2 & 2 & 2 & $\sim$ & 2 & 2 & 2 & 2 & 2 & $\frac{5}{2}$ & $<$ & 2 & 2 & 2 & 2 & 2 & $\stackrel{\rho}{\infty}$ & 2 & 2 & 2 \\
\hline ய..оร วુ!า & 志 & $\Xi$ & $\overleftarrow{\Xi}$ & $\Xi$ & $=$ & $\simeq$ & $工$ & $=$ & $\stackrel{60}{=}$ & $=$ & $\Sigma$ & - & 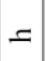 & $工$ & $\Xi$ & $工$ & $工$ & $工$ & of & 00 & 00 \\
\hline 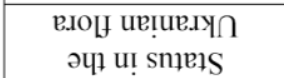 & $\ddot{\sim}$ & $\ddot{v}$ & $\ddot{v}$ & $z^{n}$ & $z^{n}$ & $z^{n}$ & $z^{n}$ & $z^{n}$ & $\stackrel{\pi}{=}$ & $z^{n}$ & $z^{\infty}$ & $\cong$ & $z^{n}$ & $\stackrel{\Xi}{=}$ & $\ddot{v}$ & $\stackrel{\Xi}{=}$ & $z^{n}$ & $\ddot{v}$ & $z^{n}$ & $z^{n}$ & $z^{n}$ \\
\hline dno.ıô [ยuo!̣๖un & $\approx$ & $\circlearrowleft$ & ৩ & 0 & $\cong$ & $\cong$ & $\cong$ & $\cong$ & ৩ & $\cong$ & $\stackrel{\Omega}{=}$ & $\circlearrowleft$ & $\circlearrowleft$ & $\cong$ & $\circlearrowleft$ & $\circlearrowleft$ & $\cong$ & $\circlearrowleft$ & $\cong$ & $\cong$ & 此 \\
\hline 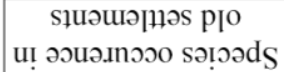 & $\bar{\sim}$ & $\infty$ & - & - & $\infty$ & - & $\infty$ & $\simeq$ & 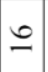 & $N$ & - & 으 & + & $\infty$ & in & $\infty$ & $N$ & - & $\cong$ & 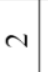 & 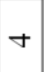 \\
\hline 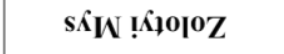 & 그 & & & & & & & & $\sim$ & & & $N$ & & $m$ & & & & & $\sim$ & & \\
\hline 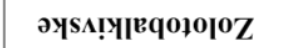 & 2 & $N$ & & & & - & $m$ & & $\sim$ & - & & $m$ & - & $N$ & & - & & & & & - \\
\hline วУSU & $\infty$ & & & & - & & - & $N$ & $m$ & & & & & $N$ & & & & & - & & $N$ \\
\hline 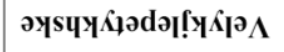 & $\Xi$ & $N$ & - & & & & $\sim$ & $N$ & $N$ & & & $N$ & & $m$ & $N$ & - & & & & & \\
\hline 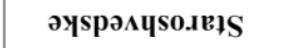 & $\underline{-}$ & - & & & $N$ & & & & - & & & $\sim$ & & $N$ & N & - & & & - & & - \\
\hline әуSSAE[S!UE]S & $\cong$ & & & & & & & - & $N$ & & & & & $m$ & & & & & $N$ & $N$ & \\
\hline вУІәУS & $\Xi$ & & & & & & & $\sim$ & N & & & & - & $m$ & & & & & $\sim$ & - & \\
\hline әуss!yniqus & $\cong$ & & & & $N$ & & & $N$ & - & & & - & & $N$ & $N$ & - & & & - & & \\
\hline 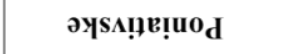 & $\simeq$ & & & & - & & & & & & & $\sim$ & - & $N$ & & 一 & & - & - & & \\
\hline 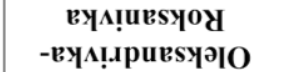 & $=$ & & & - & & & & & - & & - & - & & $N$ & & - & - & & - & & \\
\hline 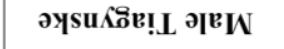 & 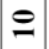 & $\sim$ & & & & & & $\sim$ & - & & & & & $m$ & & & & & & & \\
\hline 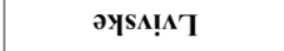 & $a$ & - & & & $\sim$ & & - & - & - & & & - & - & $\sim$ & & - & & & $N$ & & \\
\hline 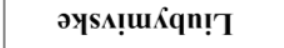 & $\infty$ & $N$ & & & $N$ & & & - & - & & & $N$ & & $N$ & & & & & & & \\
\hline әу[SA!jnsuoy & $r$ & & & & - & & - & $\sim$ & $N$ & - & & & & $m$ & & & & & $N$ & & \\
\hline 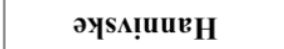 & 0 & - & & & & & - & - & & & & & & $m$ & & & & & & & \\
\hline 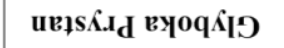 & in & & & & & & - & & $N$ & & & & & $m$ & & - & & & $N$ & & \\
\hline әУ[SA![K.IAED & $\nabla$ & & & & & & $N$ & - & $N$ & & & $N$ & & $N$ & - & & & & - & & \\
\hline 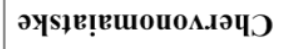 & $m$ & - & & & - & & & - & $N$ & & & & & - & - & & - & & - & & - \\
\hline 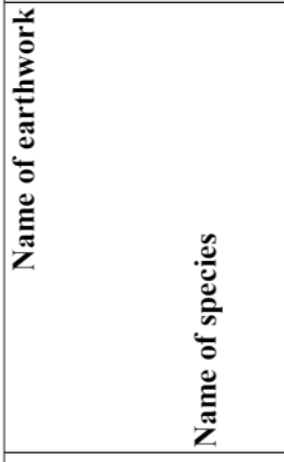 & $N$ & 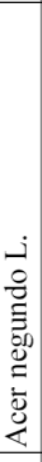 & 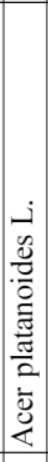 & 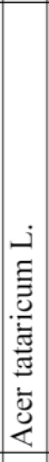 & 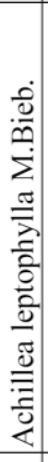 & 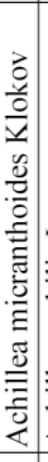 & 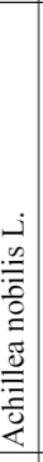 & 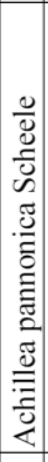 & 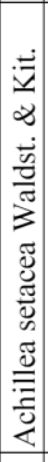 & 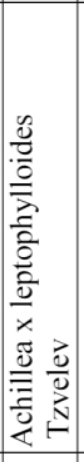 & 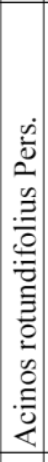 & 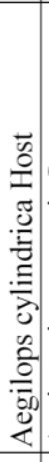 & 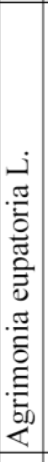 & 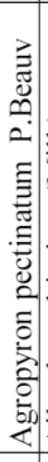 & 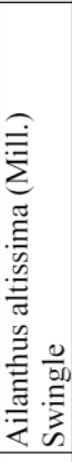 & 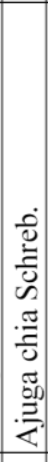 & 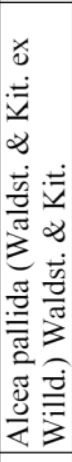 & 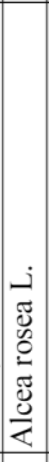 & 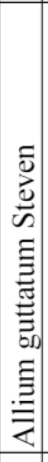 & 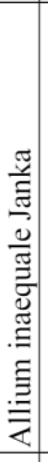 & 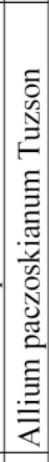 \\
\hline 米 & - & - & $\mathrm{i}$ & $\dot{m}$ & $\dot{+}$ & $\dot{n}$ & ن & $r^{\circ}$ & $\dot{\infty}$ & $\sigma^{\circ}$ & $\dot{0}$ & $\dot{=}$ & ¿ & $\ddot{2}$. & \pm & 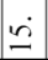 & $\stackrel{0}{0}$ & \pm & $\stackrel{\infty}{0}$ & 2 & $\dot{\sim}$ \\
\hline
\end{tabular}




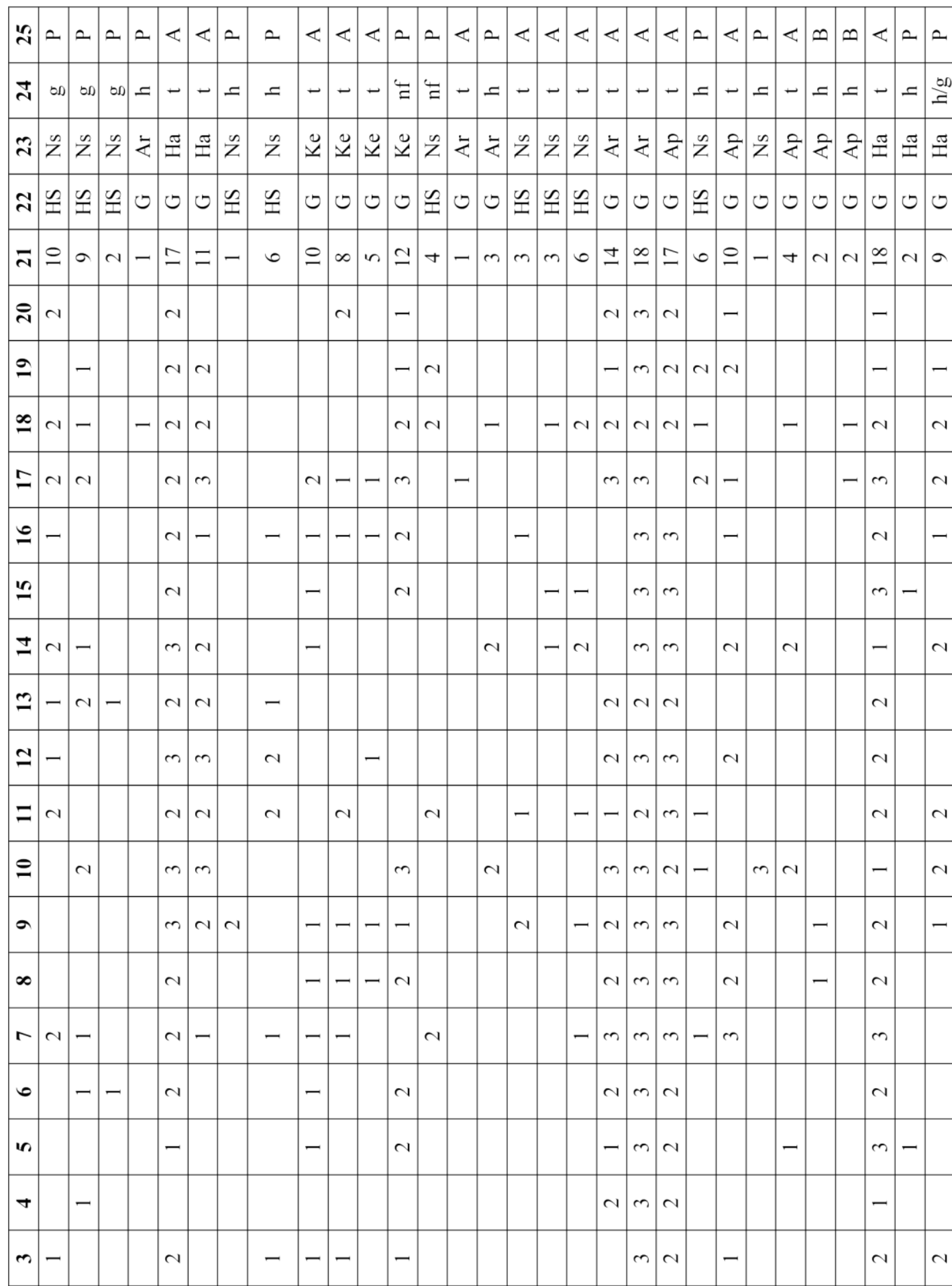

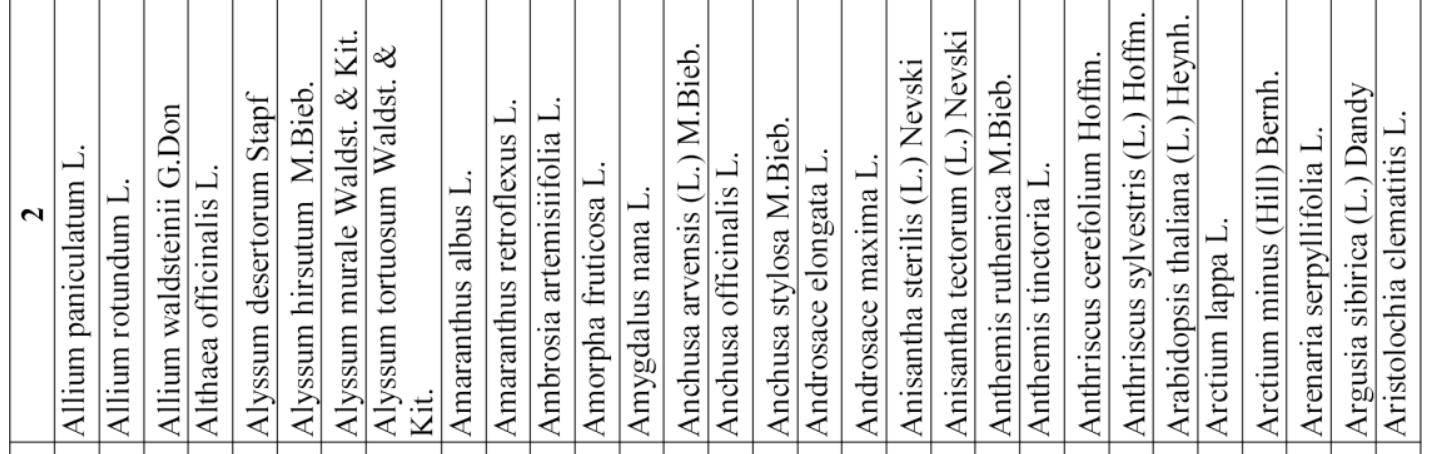

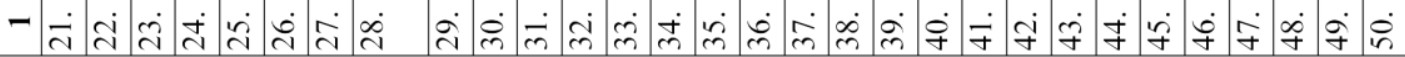


Moysiyenko I.I., Dayneko P.M., Sudnik-Wójcikowska B., Dembicz I., Zachwatowicz M., Zakharova M.Ya.

\begin{tabular}{|c|c|c|c|c|c|c|c|c|c|c|c|c|c|c|c|c|c|c|c|c|c|c|c|c|c|c|}
\hline 2 & 2 & 20 & 2 & 2 & a & 2 & $\infty$ & 2 & 2 & 2 & 2 & 2 & $\ll$ & 2 & 2 & 2 & 2 & 2 & 2 & 2 & 20 & 2 & $a$ & 2 & 2 & $<$ \\
\hline $\overrightarrow{\mathrm{N}}$ & 岁 & 0 & 0 & $=$ & 0 & 0 & 5 & 0 & $\cong$ & on & on & or & - & $=$ & $=$ & 0 & $=$ & $=$ & $=$ & $=$. & $=$ & $\cong$ & $=$ & $\cong$ & $=$ & - \\
\hline 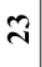 & $\ddot{\simeq}$. & $\dot{z}=$ & $\stackrel{\mathbb{I}}{\Psi}$ & $\stackrel{\mathbb{I}}{\mathbb{I}}$ & $\bar{z}$ & $z$ & दे & $z$ & दे & z & $z^{n}$ & $z^{n}$ & \& & z & z & $\tilde{z}$ & $z^{n}$ & 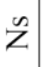 & $z^{n}$ & $z=$ & $\tilde{z}$ & $z$ & $z^{n}$ & $z^{n}$ & $\tilde{z}$ & $\ddot{\Perp}$ \\
\hline$\approx$ & 0 & ט & ט & $\cong$ & ט & 0 & 0 & $\cong$ & ט & $\cong$ & ט & 0 & ט & $\varrho$ & $\cong$ & $\cong$ & $\cong$ & $\cong$ & $\mathscr{A}$ & $\cong$ & $\cong$ & $\cong$ & $\cong$ & $\cong$ & $\cong$ & ט \\
\hline $\bar{\sim}$ & $=$ : & $\cong 9$ & $\infty$ & $I$ & + & - & in & - & ナ & 0 & $N$ & $a$ & ナ & 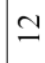 & 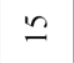 & $m$ & $m$ & - & - & in & $\circ$. & $\nabla$ & in & $a$ & $\sim$ & - \\
\hline$\stackrel{\sim}{*}$ & & & $m$ & $m$ & & & & & - & & & & - & $\mathrm{N}$ & - & & & & & - & & - & & & & \\
\hline 2 & - & -1 & $m$ & - & & & & & & - & & $N$ & - & a & 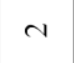 & & - & - & & & $m$ & & - & & - & \\
\hline$\stackrel{\infty}{\simeq}$ & - & $-c$ & $m$ & - & & & & & & & & N & & - & - & - & & & & & - & $N$ & - & & - & \\
\hline$\Xi$ & $\sim \Omega$ & $\begin{array}{lll} \\
N\end{array}$ & $m$ & $N$ & & & & & $\alpha$ & - & & & & - & - & & & & & & $N$ & & & & & \\
\hline$\stackrel{\bullet}{2}$ & & & $m$ & $N$ & & & & & & & - & & & & $N$ & & & & & & & & & - & & \\
\hline$\because$ & - & -1 & $m$ & $m$ & - & & $\mathrm{N}$ & & - & & & & & - & $N$ & & & & & $\sim$ & & - & & & & \\
\hline$\Xi$ & & $-c$ & $m$ & $N$ & & & $N$ & & & & & & - & & & & - & & &.- & - & & & - & & \\
\hline 2 & & $-c$ & $m$ & $N$ & & & & & & & & N & & N & - & & & & - & & - & & - & - & & \\
\hline$\simeq$ & & & $m$ & $N$ & & & & $N$ & & & & & & - & $N$ & & & & & & & & & - & & \\
\hline$=$ & - & $-\sigma$ & $m$ & - & - & - & & & & & & & & - & N & - & & & & - & - & & - & & & \\
\hline$\varrho$ & & $N=$ & $m$ & & & & & & & - & & 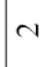 & & - & $\alpha$ & & & & & & & & & & & \\
\hline$a$ & & $m s$ & $N$ & $N$ & & & - & & & & & - & - & & $N$ & & & & & & - & & & & & \\
\hline$\infty$ & $-c$ & $\begin{array}{lll} & \end{array}$ & $m$ & $n$ & & & $\mathrm{~N}$ & & & & & & & - & $N$ & & & & & & - & & & $N$ & & $N$ \\
\hline$r$ & - & & $m$ & $N$ & $N$ & & & & & - & - & $N$ & & - & $N$ & - & & & & & - & & - & - & & \\
\hline 0 & - & $-c$ & $m$ & $N$ & & & & & & - & & - & & - & & & & & & & & & & - & & \\
\hline in & - & & $m$ & $m$ & & & & & - & & & & & & & & - & & & - &.- & - & & & & \\
\hline $\boldsymbol{\nabla}$ & - & -1 & $m$ & - & - & & $N$ & & & & & $\sim$ & & & - & & & & & & & & & - & & \\
\hline$m$ & -- & $-\sigma$ & $m$ & $\sim$ & & & & & & - & & - & & & - & & & & & & & & & - & & \\
\hline$N$ & 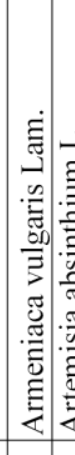 & 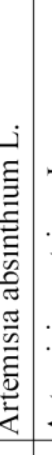 & 思 & 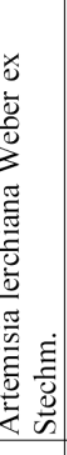 & 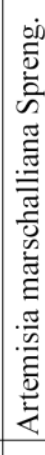 & 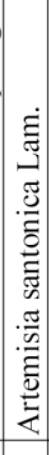 & 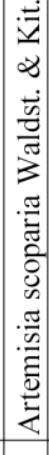 & 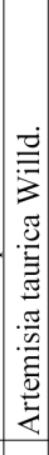 & 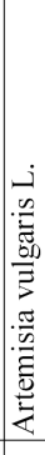 & 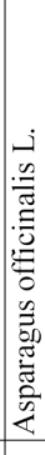 & 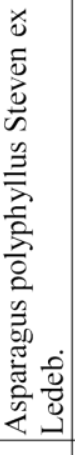 & 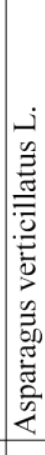 & 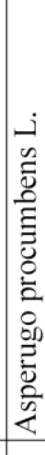 & 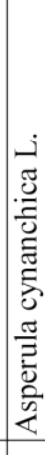 & 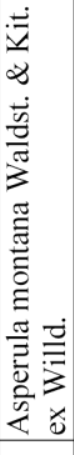 & 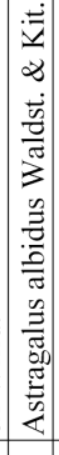 & 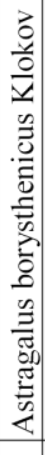 & 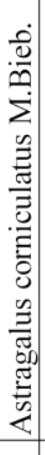 & & 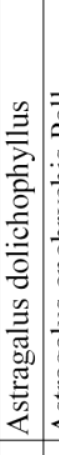 & 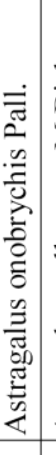 & 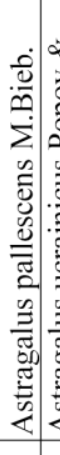 & 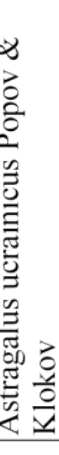 & & 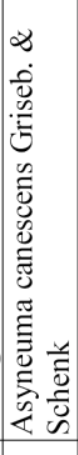 & 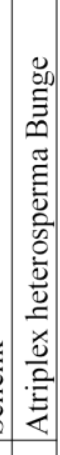 \\
\hline & & & & & & & & & & & & & & & & & & & & $\dot{R}$ & & & & & & \\
\hline
\end{tabular}




\begin{tabular}{|c|c|c|c|c|c|c|c|c|c|c|c|c|c|c|c|c|c|c|c|c|c|c|c|c|c|}
\hline 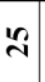 & $\ll$ & $\ll$, & $<$ & $<$ & $<$ & $\omega$ & $\ll$ & $<$ & 2 & 2 & $\sum^{\infty}$ & $a$ & $a$ & 2 & 2 & $\sum_{1}^{n}$ & $<$ & $\ll \theta$ & 2 & $\varangle$ & $a$ & 2 & $<<$ & & $\infty$ \\
\hline$\vec{\sim}$ & - & - & - & - & - & $=$ & - & - & का & $\Xi$ & $\Sigma$ & $=$ & $=$ & $=$ & on & 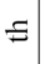 & - & - & o & - & $=$ & 象 & - & $-=$ & $==$ \\
\hline$\ddot{\sim}$ & $\stackrel{\mathbb{I}}{ \pm}$ & ২े & $\dot{<}$. & $\dot{\varepsilon}$ & $\underline{v}$ & $\dot{4}$ & 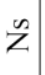 & $\stackrel{\mathbb{I}}{ \pm}$ & $z$ & $z$ & दे & z & $z^{n}$ & $\stackrel{\Xi}{ \pm}$ & $z^{n}$ & ২े & $\stackrel{\pi}{=}$. & $\dot{<}$ & $\ddot{v}$ & $\dot{<}$ & दे & $\mathbb{\pi}$ & & & $z$ \\
\hline ส & ט & ט & ( & ט & ט & 0 & ט & ৩ & $\stackrel{a}{ \pm}$ & 0 & ט & $\cong$ & $\cong$ & ט & $\cong$ & ט & ن & ن & 0 & ט & $\bullet$ & o & & (ט) & $c \cong$ \\
\hline $\bar{\sim}$ & $=$ & $m$. & + . & in & 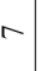 & $\bullet$ & $N$ & + & $\mathrm{N}$. & + & $N$ & - & $m$ & $a$ & $\infty$ & $m$ & -9 & $\infty$ & $N \stackrel{0}{N}$ & $\stackrel{\infty}{-}$ & $\sim$ & + & & $m r$ & - \\
\hline సิ & $N$ & & & $m$ & $N$ & & & & & & & & & & & & & $N$ & & $N$ & & - & & & \\
\hline 2 & & & - & & & - & & & - & & & & & & $N$ & & & $N$ & & - & & & & - & \\
\hline$\stackrel{\infty}{\infty}$ & - & & - & & - & $N$ & & & - & - & & & $N$ & $N$ & $\sim$ & & & $N$ & & $N$ & & - & & $\sim$ & \\
\hline$\simeq$ & - & $\sim$ & & & & $N$ & & & & & & & & & $n$ & & & $m$ & & N & & & & & \\
\hline$\stackrel{0}{-1}$ & - & & & & - & $N$ & & - & & & & & & & & & & $N$ & & $N$ & & - & & - & \\
\hline$\because$ & - & & - & $m$ & - & & & $\mathrm{N}$ & & & & & & & & - & & $m$ & & N & 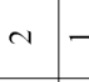 & - & & & \\
\hline \pm & & & & $N$ & & $\mathrm{~N}$ & - & & & & - & & & $m$ & & & & $m-$ & - & $N$ & & 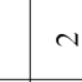 & & - & \\
\hline$\cong$ & & & & & & $N$ & & & & & & $N$ & - & $N$ & n & & & $m$ & & $N$ & & - & & - & \\
\hline$\simeq$ & $N$ & & & & & $m$ & & & & & & & & & & & & $m$ & & $m$ & & $N$ & & & \\
\hline$=$ & & &.- & - & & - & & & & & & & & $N$ & - & & & $N$ & & - & & - & & - & \\
\hline 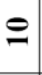 & & & & & & $m$ & & & & - & & & & $m$ & - & & & - & & $m$ & & $N$ & & & \\
\hline$a$ & $\sim$ & & & & - & $N$ & & $N$ & & & & & - & & & & & $N$ & & $m$ & & - & & & \\
\hline$\infty$ & $m$ & & & & - & $N$ & & & & & & & & $N$ & & & & $m$ & & $N$ & $N$ & - & & - & \\
\hline$r$ & - & - & & & & $N$ & & & & $N$ & $N$ & & & - & - & & -9 & $m$ & & $m$ & & $\sim$ & & & - \\
\hline ( & & & & & & $N$ & & & & & & & & $N$ & N & - & & $m$ & & - & & & & & \\
\hline n & & & & $N$ & & - & - & & & & & & & & & & & $N$ & & $N$ & & - & & & \\
\hline$\nabla$ & $N$ & & & & & $m$ & & & & & & & & $N$ & & $N$ & & $m-$ & $\begin{array}{ll}-0 \\
\end{array}$ & $N$ & & - & & & \\
\hline$m$ & - & - & & & - & - & & $N$ & & - & & & & & & & & $N$ & & $N$ & & - & & & \\
\hline$N$ & 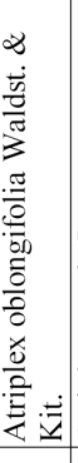 & 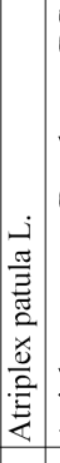 & 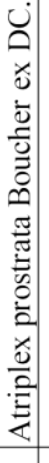 & 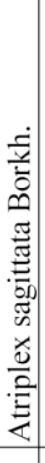 & & 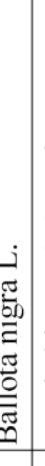 & 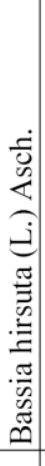 & 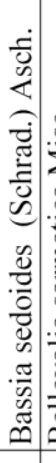 & & 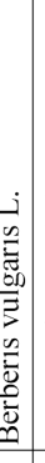 & & 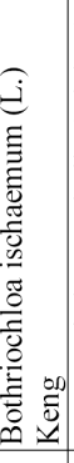 & 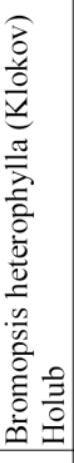 & & 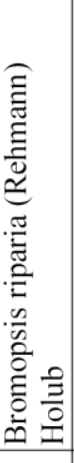 & 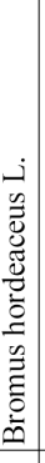 & 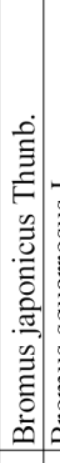 & 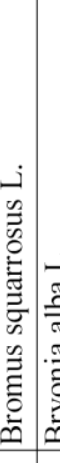 & 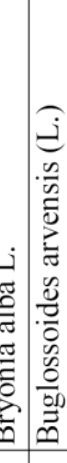 & 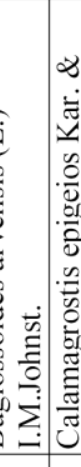 & 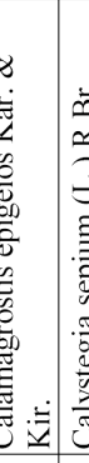 & 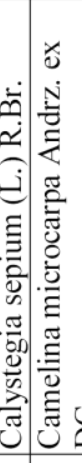 & 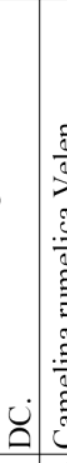 & 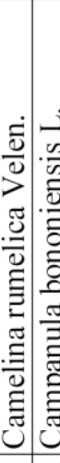 & 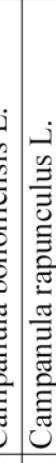 \\
\hline-1 & & 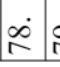 & 2 & $\dot{\infty}$ & $\dot{\infty}$ & îd: & & 官l: & & $\therefore:$ & & & & & & & & & $2 \approx$ & & & & & 8 & $\dot{0}$ \\
\hline
\end{tabular}




\begin{tabular}{|c|c|c|c|c|c|c|c|c|c|c|c|c|c|c|c|c|c|c|c|c|c|c|c|c|c|}
\hline 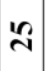 & $\infty<$ & $\frac{n}{<}$ & 2 & $\stackrel{n}{<}=$ & $\infty \xi$ & $\begin{array}{l}b \\
2\end{array}=$ & 2 & 2 & 2 & 2 & 2 & 20 & 2 & $-\frac{\mathscr{f}}{<}$ & $=$ & a & $\sim$ & 2 & $<$ & $<$ & $<$ & $=0$ & & & \\
\hline$\vec{N}$ & $=-$ & 5 & $=$ & $\mp$. & $=5$ & 5 & 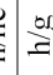 & $\frac{30}{2} \simeq$ & $\stackrel{60}{=}$ & $=$ & $\stackrel{\infty}{\geq}$ & $\stackrel{\infty}{\geq}=$ & $\sum_{\Sigma}^{\mathscr{L}}$ & $=$ & $E=$ & $=$ & $=$ & $=$ & - & - & -4 & $\vec{z}$ & & - & \\
\hline$\tilde{\sim}$ & $z \ddot{z}$ & 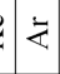 & $\ddot{\ddot{x}}$ & $\dot{z}$ & दे & $z$ & 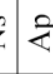 & z & $\stackrel{\underline{3}}{\underline{3}}$ & $\stackrel{\underline{J}}{\underline{I}}$ & $z$ & $z^{n}$ & $z$ & $\hat{z}$ & $z$ & $\stackrel{\Xi}{I}$ & $\cong$ & $z^{n}$ & $z^{n}$ & रे & $\stackrel{\mathbb{3}}{ \pm}$ & $z$ & & $\underline{z}$ & \\
\hline ส & $\cong 0$ & 0 & 0 & 0 & 0 & $=0$ & 0 & $\cong$ & ט & ט & $\cong$ & $\cong$ & ৩ & $E 0$ & 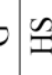 & $\cong$ & ט & $\cong$ & ט & 0 & 0 & ט ט & & כ & 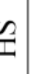 \\
\hline $\bar{\sim}$ & in $m$ & 0 & $\cong$ & $N$. & $-\cong$ & $=-$ & $N$ & $a$ & \pm & $N$ & $\because$ & $\infty \quad$, & $m$ & $\checkmark=$ & $=-$ & 0 & - & + & $\because$ & $n$ & - & $n-$ & & v & \\
\hline సิ & & - & 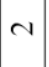 & & & & & & N & & $m$ & & & o & & & & & N & & & & & & \\
\hline 2 & - & - & - & & - & - & & $N$ & N & - & - & - & & - & & & & - & & & & & & & \\
\hline$\stackrel{\infty}{-}$ & - & - & - & & - & - & - & $N$ & $N$ & & $m$ &.- & - & $-\curvearrowright$ & & $\alpha$ & - & $N$ & N & - & - & - & & & \\
\hline 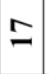 & - & - & $m$ & - & & & & & & & - & & & r & & & & & - & & & $r$ & & & \\
\hline$\stackrel{0}{0}$ & & - & & & & & & & $N$ & & $m$ & & & r & & & & & - & & & & & & \\
\hline 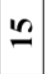 & & $m$ & - & & & & & & $\mathrm{N}$ & & $m$ & - & & r & & & & & N & & & & & & \\
\hline \pm & & $N$ & & & 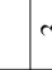 & $m$ & & $N$ & $N$ & & $m$ & & & r & & & & - & $N$ & & $N$ & & & & \\
\hline 2 & - & - & & & c & m & & $n$ & $m$ & & - & & & - & & $N$ & & & N & & & & & & \\
\hline $\mathcal{I}$ & & $\sim$ & - & & & $N$ & & & & & $m$ & $\sim$. & - & - & & & & & $m$ & & & & & & \\
\hline$=$ & & & $N$ & - & o & $v$ & & & $N$ & & $N$ & - & - & $-r$ & & 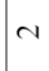 & & & N & - & & & & & \\
\hline 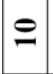 & - & $\sim$ & $\mathrm{N}$ & & & $N-$ & $-N$ & $m$ & $\mathrm{~N}$ & & & & - & r & & - & & & - & & & $N$ & & & \\
\hline$\sigma$ & - & N & $N$ & & $-c$ & $N$ & & & $\mathrm{~N}$ & & $m$ & & & o & & N & & & N & & & - & & N & \\
\hline$\infty$ & & $N$ & & & & & & & & & $m$ & & & o & & & & & N & & & & & & \\
\hline$r$ & - & $N$ & $N$ & & & - & & $\sim$ & & & & $N$ & & r & $\mathrm{N}-$ & $\mathrm{N}$ & & - & - & & & & & & \\
\hline 0 & & & & & & $m$ & & m & $N$ & - & $m$ & $N$ & & - & & & & & & & & & & & \\
\hline in & & - & $N$ & & & $N$ & & - & $N$ & & $N$ & - & & & & & & & $\sim$ & & & & & - & \\
\hline$\nabla$ & - & $N$ & - & & & $N$ & & $m$ & $N$ & & & & & - & & & & & & & & & & & \\
\hline$m$ & & - & $N$ & & & - & & & $N$ & & - & & & 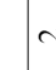 & & & & & & - & & & & & \\
\hline
\end{tabular}

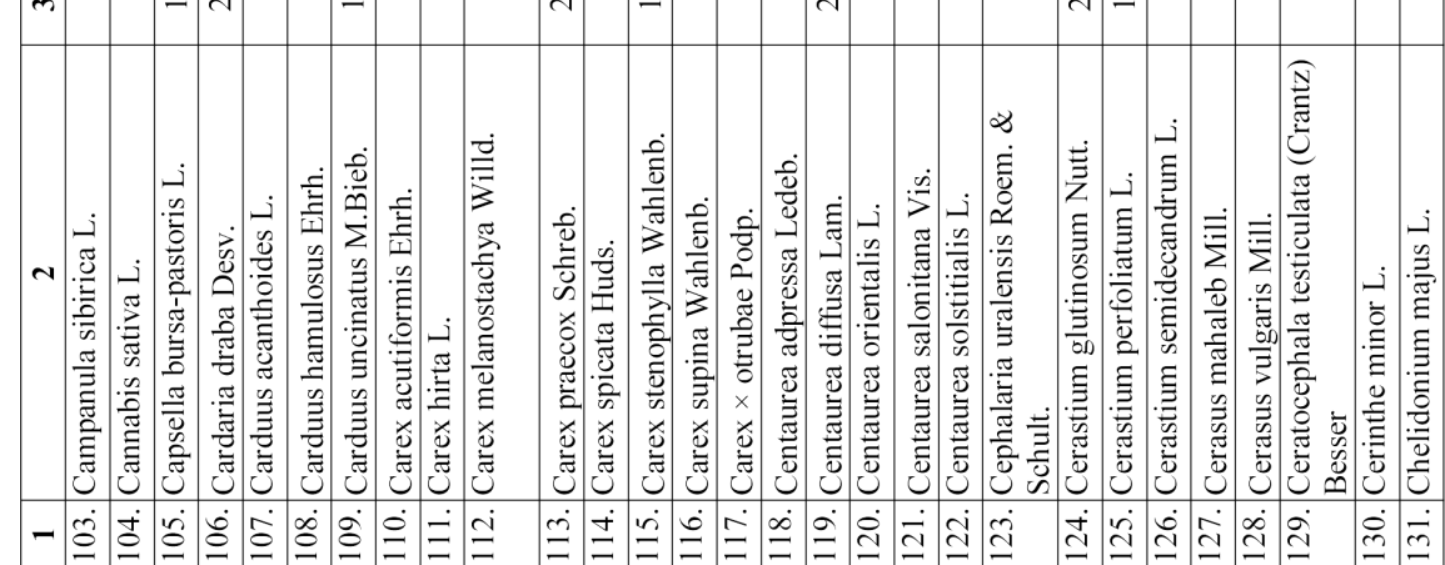




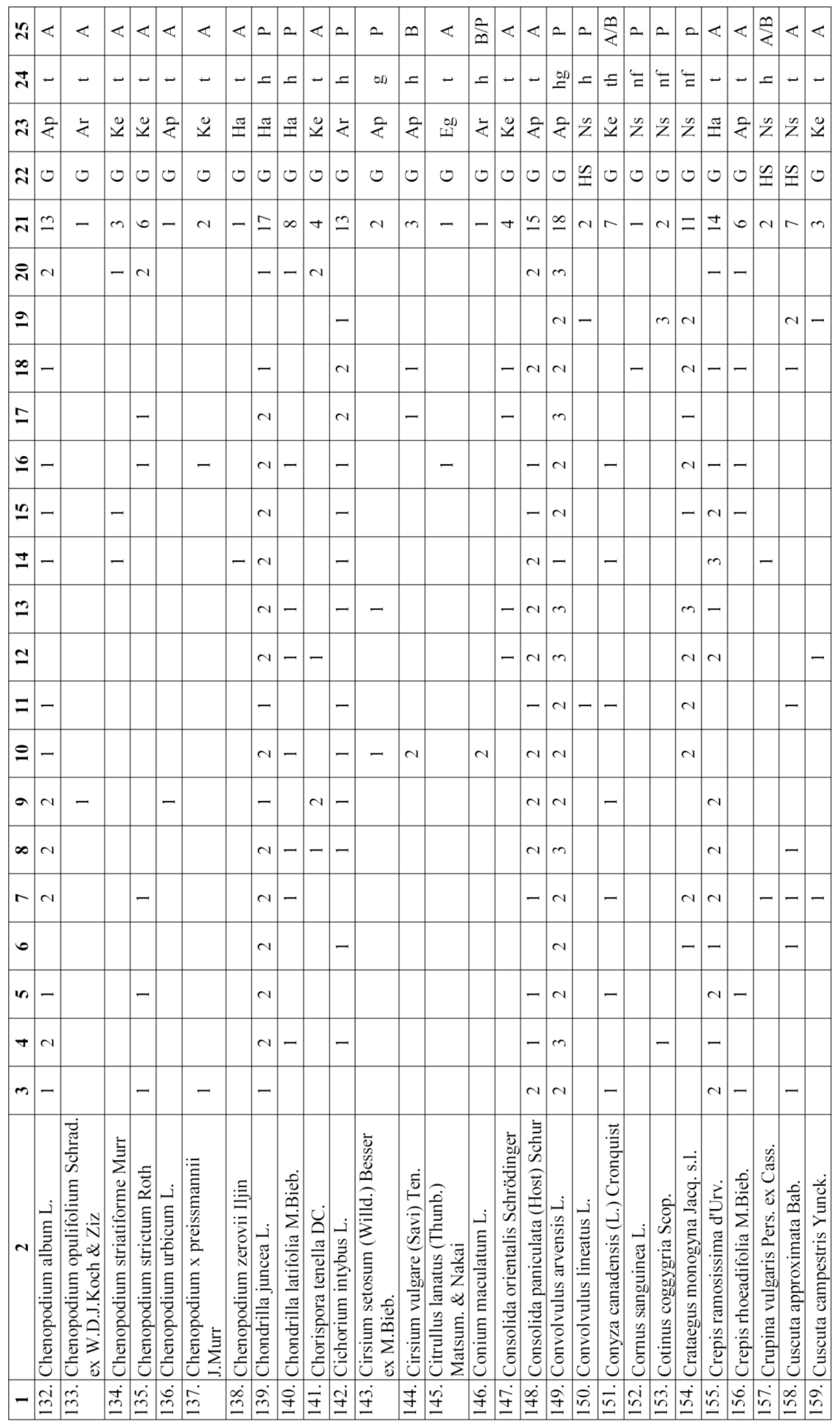


Moysiyenko I.I., Dayneko P.M., Sudnik-Wójcikowska B., Dembicz I., Zachwatowicz M., Zakharova M.Ya.

\begin{tabular}{|c|c|c|c|c|c|c|c|c|c|c|c|c|c|c|c|c|c|c|c|c|c|c|c|c|}
\hline $\mathscr{N}$ & $<$ & a & $\sim 0$ & $m=$ & $\varangle$ & $<$ & 2 & 2 & 2 & 2 & $\stackrel{\infty}{<}$ & $\ll$ & 2 & $\ll$ & 2 & $\infty$ & $\infty$ & 2 & $a$ & $\sum_{<}^{\infty} a$ & 2 & 2 & $a$ & $a$ \\
\hline$\vec{\sim}$ &.- & $=$ & $\stackrel{\infty}{\geq}=$ & $=\stackrel{0}{2}$ & & - & $=$ & $=$ & $=$ & $=$ & $\xi$ & - & $=$ & - & $=$ & $=$ & $=$ & $\vec{\Xi}$ & $=$ & 5 & & $\stackrel{00}{=}$ & $=$ & $\stackrel{\infty}{\geq}$ \\
\hline$\tilde{\imath}$ & ₹े: & $\stackrel{\pi}{ \pm}$ & दे & $\dot{<}$ & & $\dot{z}$ & $z^{n}$ & $z$ & z & $z^{n}$ & $\tilde{z}$ & 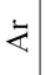 & $\ddot{v}$ & 之 & $\Xi$ & $z$ & दे & $\ddot{\ddot{v}}$ & $z^{n}$ & $z^{n}$ & $\cong$ & दे & $z^{n}$ & $z_{z}^{n}$ \\
\hline 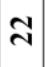 & ن & 0 & ט & ט 0 & & 0 & $\cong$ & $\cong$ & $\cong$ & 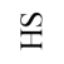 & $\cong$ & ט & ט & ט & $\cong$ & $\cong$ & | & ט & $\cong$ & ט & ט & 0 & $\cong$ & $\cup$ \\
\hline $\bar{\sim}$ & + & + & 0. & $r$ & 气 & $\underline{0}$ & - & $\cong$ & - & + & N & $N$ & $N$ & - & $m$ & $\nabla$ & $\infty$ & \pm & 0 & - & 0 & $\infty$ & $v$ & $N$ \\
\hline స్ & & & & & & $N$ & & $\sim$ & & & & & & & & & & & & & & $m$ & & \\
\hline 2 & & & & - & & - & & $N$ & & & - & & & & - & & - & - & & & - & $m$ & & \\
\hline$\stackrel{\infty}{-}$ & N & & $N$ & & & & & & & & & & & & - & - & - & $N$ & - & & $N$ & m & & \\
\hline$=$ & & & & $-\gamma$ & 0 & N & & & & & & - & & & & & & - & & & $\sim$ & $m$ & & \\
\hline$\stackrel{0}{0}$ & & & - & & & $m$ & & - & & & & & & & & & & - & & & - & N & & \\
\hline$\because$ & & & - & & & $N$ & & $\mathrm{~N}$ & & & & & & & & & & - & - & & & $m$ & & \\
\hline \pm & & & & & & $N$ & & - & & - & & & & & & & & & $N$ & & & $N$ & & - \\
\hline 2 & & - & & - & & $N$ & & - & & & & & & & & & & - & & & & $N$ & & $\sim$ \\
\hline $\mathbb{Z}$ & & & $N$ & & & $N$ & & $N$ & & & & & & & & - & $N$ & $N$ & & & & $m$ & & \\
\hline$=$ & $N$ & - & & & & $N$ & & - & & & - & - & $N$ & & & - & - & - & & - & $N$ & $N$ & & \\
\hline$\Theta$ & - & & & - & & $N$ & & & & & & & - & & - & & - & - & m & & & $m$ & & \\
\hline$a$ & & & & - & & $N$ & & & & & & & & & & - & - & & & & & $N$ & & \\
\hline$\infty$ & & & & - & & v & & $m$ & & & & & & & & & - & - & & & & $N$ & & \\
\hline$r$ & & & & - & & N & $N$ & $\sim$ & $N$ & - & & & & & & & & - & - & & - & $N$ & - & \\
\hline 0 & & & & & & $N$ & & - & & & & & & & & & & & & & & $m$ & & \\
\hline in & & $N$ & $N$ & & & $N$ & & - & & - & & & & & & & - & - & $\sim$ & & & $N$ & & \\
\hline T & & & & - & & $N$ & & & & & & & & & & & & - & & & & $m$ & & \\
\hline$m$ & - & -0 & $N$ & & & & & $\mathrm{~N}$ & & - & & & & - & & & & - & & & & $m$ & - & \\
\hline$N$ & 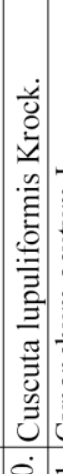 & 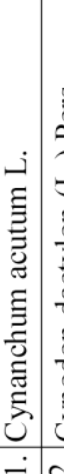 & 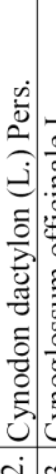 & 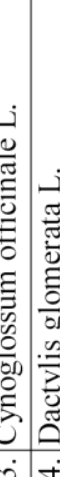 & 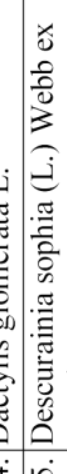 & 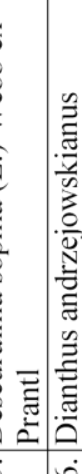 & 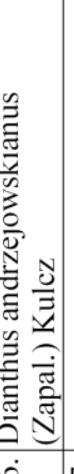 & 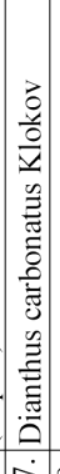 & 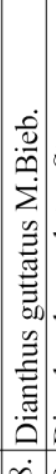 & 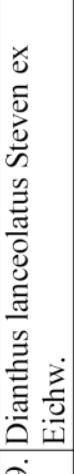 & 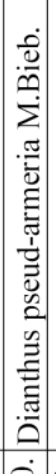 & 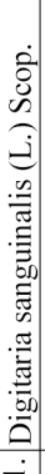 & 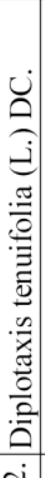 & 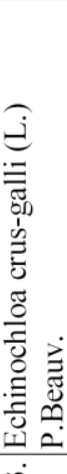 & 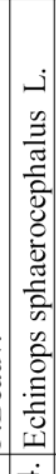 & 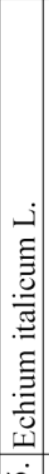 & 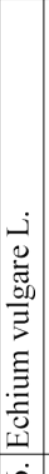 & 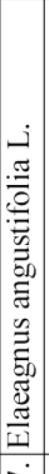 & 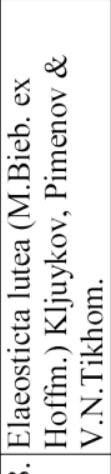 & 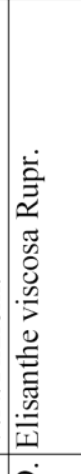 & 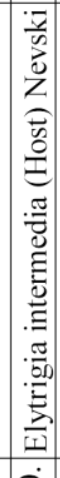 & 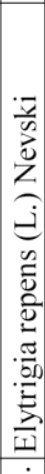 & 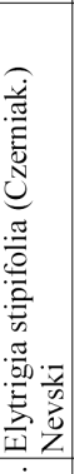 & 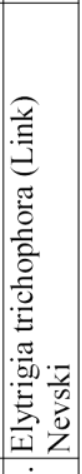 \\
\hline & 10 & -6 & రీ & 6 & & 8 & 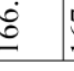 & $\underline{\hat{0}}$ & $\left(\begin{array}{c}\dot{0} \\
0 \\
-\end{array}\right.$ & & 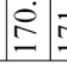 & & & & & I & $\cong$ & & & $\stackrel{2}{1}$ & $\dot{\otimes}$ & $\dot{\infty}$ & & \\
\hline
\end{tabular}




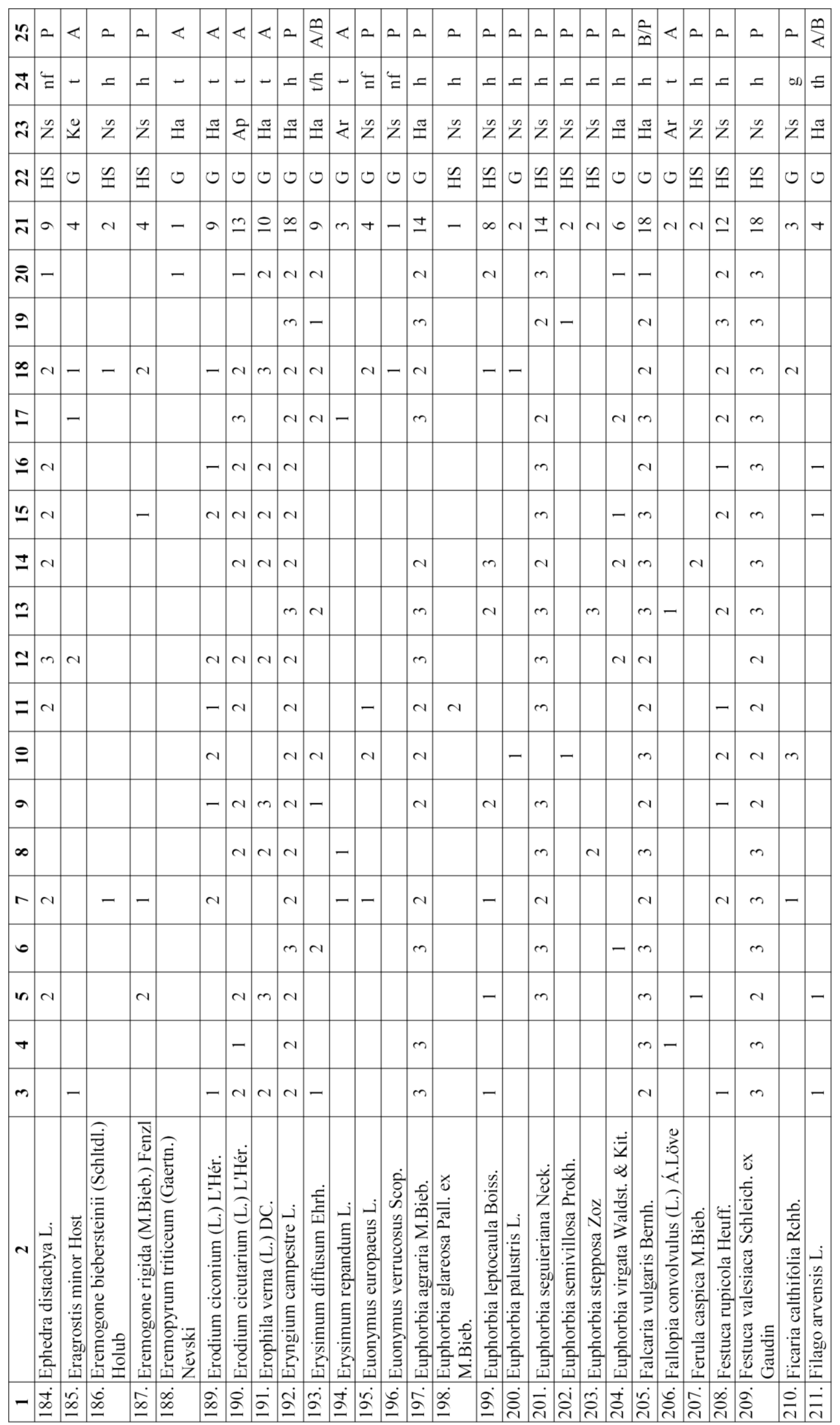




\begin{tabular}{|c|c|c|c|c|c|c|c|c|c|c|c|c|c|c|c|c|c|c|c|c|c|c|c|c|c|c|}
\hline 르 & a & 2 & a. & $\ll$ & $\varangle A$ & $=2$ & 2 & 2 & 2 & $\ll$ & 2 & $\sim=$ & $a$ & $<$ & 2 & 2 & $<$ & $\sum_{<}^{\infty}=$ & $4<$ & & $=8$ & 10 & $=$ & $a$ & 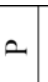 & $a$ \\
\hline$\vec{N}$ & & $\Xi$ & $\bar{\Xi}$ & - & -8 & of & תם & ס ס & $工$ & - & $\simeq$ & $=$ & $=0$ & $:-$ & $=$ & $=$ & - & $5=$ & $=$ & & क्षा & & $==$ & $=$ & $=$ & of \\
\hline$\approx$ & & & $\underset{ \pm}{ \pm}$ & $\dot{z}$ & $\dot{2}$ & $z$ & $z^{n}$ & $z^{n}$ & $z$ & दे & $\stackrel{\mathbb{Z}}{\mathbb{I}}$ & $\Xi$ & $z$ & $\dot{4}$ & $\cong$ & $z$ & $\stackrel{\Xi}{\Xi}$ & $\dot{z}=$ & $\xi$ & & $\Xi$ & $z$ & & $\ddot{z}$ & $\ddot{\ddot{v}}$ & $z^{n}$ \\
\hline$\approx$ & & ט & •| & b & 0 & $\hat{1}=$ & 生 & $\bumpeq$ & $\cong$ & ט & ๑ & ن & $\underline{\underline{n}}$ & ט & 0 & $\cong$ & ن. & u| ن & כ & & ن & ט| ט & & $\mathscr{1}$ & ט & $\cong$ \\
\hline & & & & & & e & & 6 & & & 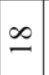 & - & & - & & $\cong$ & -5 & $\simeq$ & V & & N & & & - & + & - \\
\hline
\end{tabular}

จิ

2

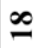
N-

$=$

는

n

$\Xi$

9

2

บ

$=-\quad+\quad-$

인

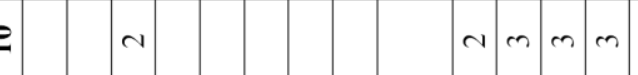

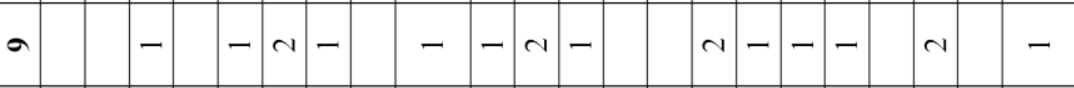

\begin{tabular}{|l|l|l|l|l|l|l|l|l|l|l|l|}
\hline$\infty$ & 4 & 4 & 4 & & & 4 & $m$ & & - & -4 & \\
\hline
\end{tabular}

$r-$

$-4$

$\operatorname{mon} 2$

$4-4-4 \mid 4$

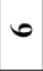

is

$-$

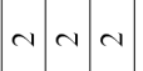

$-4$

in

$\cos$

$-4 \quad 4$

$+$

$m-$

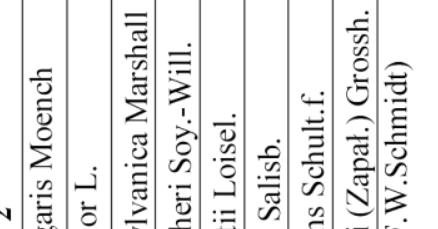

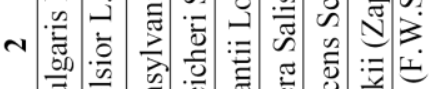

$-m m$

$--$

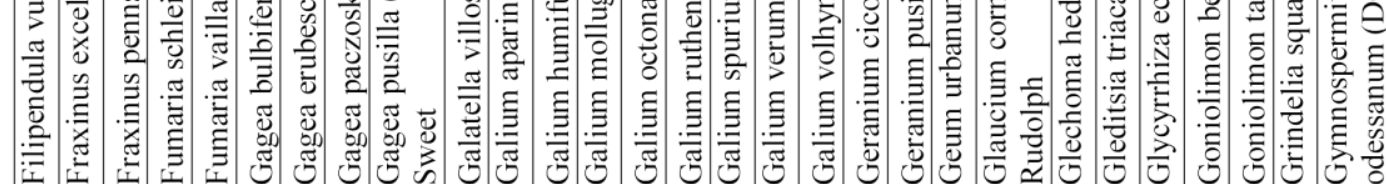

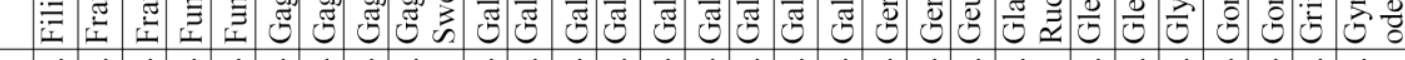

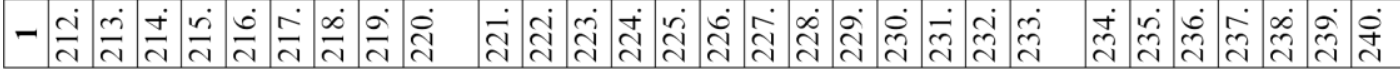




\begin{tabular}{|c|c|c|c|c|c|c|c|c|c|c|c|c|c|c|c|c|c|c|c|c|c|c|c|c|c|}
\hline $\mathscr{\sim}$ & $a$ & 2. & $\ll$ & $a$ & $\infty$ & $a$ a & 2 & 2 & $<$ & & $<2$ & 2 & $a$ & $\sum_{<}^{n}$ & 2 & 2 & 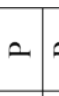 & 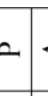 & $<1=$ & 2 & $\stackrel{\infty}{\infty}$ & & $c=$ & & 2 \\
\hline$\vec{N}$ & $\Xi$ & $=$ & - & $=$ & $=$ & $=$ & $=$ & on & - & - & $-=$ & 80 & $=$ & 5 & $=$ & $=$ & & $\begin{array}{l}\infty 01 . \\
\Sigma\end{array}$ & -0 & מo & $=$ & $=-$ & $-\leftrightarrows$ & & $=$ \\
\hline$\vec{\pi}$ & 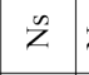 & $z=$ & $\varepsilon^{2}$ & $z^{n}$ & $\ddot{z}$ & $z^{n}$ & $z^{n}$ & $z$ & $\stackrel{\mathbb{3}}{ \pm}$ & $\stackrel{\pi}{\pi}$ & $\dot{\Xi} \cong$ & z & $z^{n}$ & $\dot{k}$ & $z^{n}$ & $\stackrel{\Xi}{=}$ & $\stackrel{\Xi}{ \pm}$ & z: & $\Xi \check{z}$ & {$\left[\begin{array}{c}\infty \\
0 \\
-1\end{array}\right.$} & $\cong$ & & $\vec{v}$ & & $z$ \\
\hline ส & $\cong$ & $\cong$ & ט & $\cong$ & $\cong$ & $\cong$ & $\cong$ & ט & 0 & ن & ט ט & $\mathscr{Z}$ & $\cong$ & ن & $\cong$ & 0 & ט & 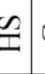 & ט & 0 & ن. & 0 0 & D & & $\mathscr{Z}$ \\
\hline $\bar{\sim}$ & $\infty$ & in & 0 & $\subseteq$ & - & +0 & $N$ & $N$ & \pm & $-s$ & $\mathbb{N} \sim$ & $m$ & 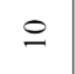 & 0 & $\because$ & $N$ & - & - & $-\nabla$ & - & - & $m-$ & $+\infty$ & & 0 \\
\hline సิ & & & $N$ & - & & & & & $m$ & & & & & & & & & & & & & & -- & & \\
\hline 2 & m & $\sim$ & & $N$ & & & - & & & & $N$ & & - & & - & & & & & & & & - & & $m$ \\
\hline 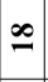 & - & $N$ & & & & - & - & $N$ & $\mathrm{~N}$ & -- & - & $m$ & - & & $N$ & & $N$ & & $\leadsto$ & & & & & - & \\
\hline$\Xi$ & & & $N$ & - & & - & & & $m$ & & $m-$ & & $N$ & & $N$ & & & & - & & & & $-N$ & & - \\
\hline$\stackrel{\bullet}{2}$ & & & & & & & & & $m$ & & $m$ & & & & - & & & & & & & & - & & \\
\hline$\cong$ & & - & - & & & & & & $m$ & & & & $\sim$ & - & & & & & & - & & & -- & & \\
\hline$\Xi$ & & - & & & & - & & & $N$ & & & & - & - & - & $\sim$ & & & 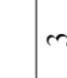 & & & & & & \\
\hline 2 & $m$ & & & $N$ & & & & & & & & & & & $N$ & & & & & & & & & & N \\
\hline $\mathbf{\simeq}$ & - & $\sim$ & - & $N$ & & & & & $m$ & & $N$ & & & & $m$ & & & & & & & & & & N \\
\hline$=$ & - & & & - & & & & & $N$ & & $N$ & & - & - & - & & & & & & & & - & & \\
\hline$\varrho$ & & & & & & & & $\sim$ & $\mathrm{N}$ & & & & $N$ & & - & & & & & & -0 & $N$ & & & N \\
\hline$a$ & - & & - & $N$ & & & & & $m$ & & $N$ & & & - & - & & & & & & & -- & & & \\
\hline$\infty$ & & & & & & & & & $m$ & & $m-$ & & - & & & & & & & & & & N & & \\
\hline$r$ & & & & - & - & - & & & $m$ & & & - & $N$ & & - & & & - & $r$ & & & & & & \\
\hline 0 & - & & & - & & & & & & & & & & - & $N$ & & & & & & & & & & N \\
\hline 10 & & & & - & & & & & $m$ & & & & - & & - & - & & & $r$ & & & & & & \\
\hline$\nabla$ & - & & & & & & & & & & $N$ & & & & $N$ & & & & & & & & & & \\
\hline$m$ & & & - & & & & & & $N$ & & $N$ & - & & - & - & & & & & & & & & & \\
\hline$\sim$ & 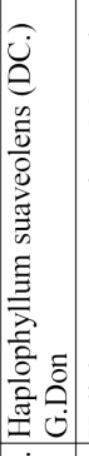 & 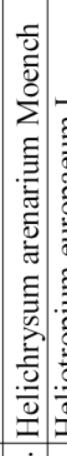 & 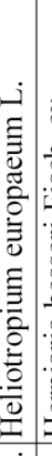 & 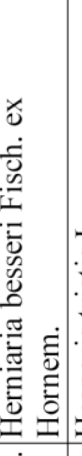 & 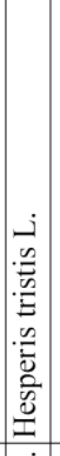 & 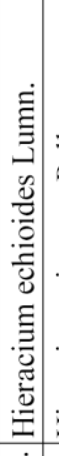 & 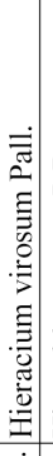 & 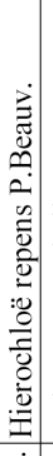 & 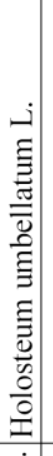 & 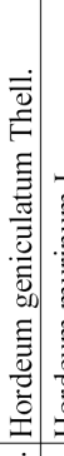 & ذُ & 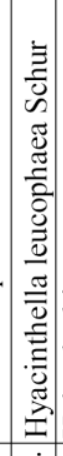 & 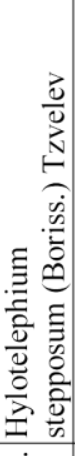 & 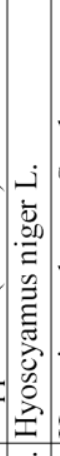 & 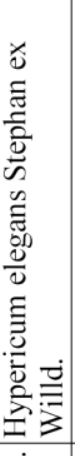 & 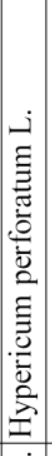 & 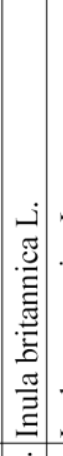 & . & 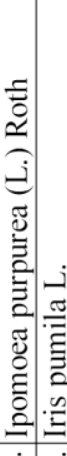 & 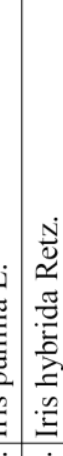 & 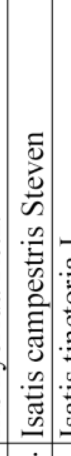 & 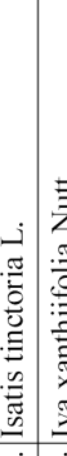 & 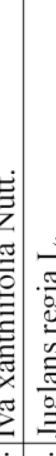 & 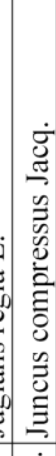 & 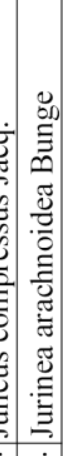 \\
\hline & & a' & & & $\ddot{q}$ & & & & & & & & & & & & $\infty$ & & & & & & & & \\
\hline
\end{tabular}


Moysiyenko I.I., Dayneko P.M., Sudnik-Wójcikowska B., Dembicz I., Zachwatowicz M., Zakharova M.Ya.

\begin{tabular}{|c|c|c|c|c|c|c|c|c|c|c|c|c|c|c|c|c|c|c|c|c|c|c|c|c|c|}
\hline 2 & 2 & $2=$ & $=2$ & $<$ & $\sim=$ & 2 & $\sum_{<}^{\infty}$ & 2 & $\ll$ & $\stackrel{n}{<}$ & $\stackrel{\infty}{<}$ & $\sum_{<}^{\infty}$ & $=0$ & & 2 & 2 & $\stackrel{n}{<}$ & $\stackrel{m}{<}=$ & $2=$ & 2 & $\sim=$ & $=2$ & 2 & 2 & 2 \\
\hline$\vec{\sim}$ & $=$. & $==$ & $=0$ &.- & $=-$ & $==$ & $\Sigma$ & $\stackrel{+0 .}{\geq}$ & - & $\xi$ & $\xi$ & 5. & $=0$ & & $==$ & $=$ & 5 & 5 & $\Xi$ & $==$ & $=$ & $==$ & $==$ & & $=$ \\
\hline$\approx$ & $z=$ & $\bar{z} \tilde{z}^{n}$ & 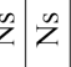 & $\ddot{\check{x}}$ & $\tilde{z}$ & $z$ & $<$ & $\stackrel{2}{<}$ & $\dot{z}$ & $\dot{z}$ & $\ddot{\square}$ & $\dot{z}$ & $z$ & & 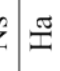 & $\stackrel{\mathbb{I}}{ \pm}$ & $\dot{z}$ & $\sum=$ & $\stackrel{\pi}{ \pm}$ & $z \quad z^{n}$ & $\tilde{z}$ & 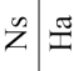 & $\stackrel{\Xi}{=}$ & & $\stackrel{\Xi}{\Psi}$ \\
\hline$\approx$ & $\cong$ & $\cong \cong$ & $2 \cong$ & 0 & $\cong$ & $\mathscr{1}=2$ & 0 & 0 & 0 & 0 & 0 & 0 & ט & 0 & 0 & 0 & 0 & ט & 0 & $\approx \Omega$ & $\cong$ & $\cong 0$ & 0 & $\cong$ & 0 \\
\hline $\bar{\tau}$ & a. & -- & $-\infty$ &.+ & -7 & $=-$ & $=$ & $r$ & $\because$ & $N$ & 인 & $\mathrm{N}$. & $-m$ & 0 & $0-$ & $\mathrm{N}$ & 0 & -0 & $\infty \curvearrowright$ & $\mathrm{N}$ & in 6 & $0=$ & $y=$ & - & 4 \\
\hline సิ & & & $m$ & & & $N$ & $N$ & $\sim$ & $N$ & & - & & & & & & $\sim$ & & & & & N & v & & \\
\hline 2 & & & $m$ & & & $m$ & & - & & & & & $r$ & & & & & & - & & & N & $4-$ & & \\
\hline$\stackrel{\infty}{-}$ & $N$ & & $N$ & & & $m-$ & $N$ & & $m$. & - & & - & & - & & & & $-c$ & $N-$ & & $N$ & $m N$ & $4-$ & - & 4 \\
\hline$\Xi$ & & & $m$ & & & $N$ & 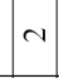 & $\sim$ & $\sim$ & & - & & - & & & & & & & & & N & $\checkmark N$ & & \\
\hline$\stackrel{0}{0}$ & & & $m$ & - & & $m$ & 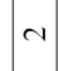 & - & $\sim$ & & $N$ & & & & & & & & & - & & & & & \\
\hline$\because$ & - & & $\sim$ & & & $N$ & $N$ & - & $\sim$ & & $N$ & - & & & & & & & & & - & 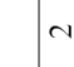 & & & \\
\hline \pm & $N$ & & $N$ & - & & $m$ & $\alpha$ & & $m$ & & - & & & & & & - & & & & $N-$ & -- & - & & \\
\hline$\cong$ & - & & $m$ & & & $N$ & $\sim$ & & & & & & & - & & & - & & $N$ & & & N & $\checkmark N$ & & \\
\hline$\simeq$ & & & $m$ & & & $N$ & $N$ & & $m$ & & & & & & & & & & - & & & & & & \\
\hline$=$ & - & & $N$ & $-c$ & $\sim \sim$ & $N$ & $\sim$ & & $N$ & & - & & & & & & N & & - & & & -- & -- & & \\
\hline 气 & - & & $m$ & & & & N & & $m$ & $\sim$ & - & & - & - & & - & & & $\sim$ & & & $m \sim N$ & v & & N \\
\hline$a$ & & & $N$ & & & $N$ & $\sim$ & & $\sim$ & & - & - & & & - & & - & & & & & & - & & \\
\hline$\infty$ & & & $m$ & $N$ & & $N$ & - & & $m$ & & & - & & - & & & & & & & & 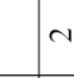 & & & \\
\hline$r$ & - & -- & $-N$ & & & $m$ & $\sim$ & & - & & $N$ & & - & & & - & & & & - & $-c$ & $N$ & - & & \\
\hline 0 & & & $m$ & & & $N$ & $N$ & - & - & & & - & & & & & & & & & & - & N & & \\
\hline in & $\sim$ & & $m$ & & & $m$ & N & - & $\sim$ & & & - & & & & & $\sim$ & & & & 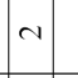 & & $1-$ & & \\
\hline$\nabla$ & & & $N$ & & & N & $N$ & & & & & & & - & & & & & - & & & & - & & \\
\hline$m$ & $N$ & & 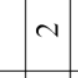 & & & $m$ & $N$ & & $\sim$ & & - & - & & - & & & & & - & & & - & - & & \\
\hline$N$ & 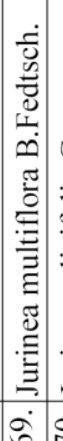 & 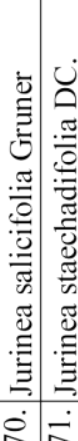 & 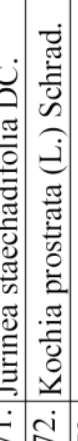 & 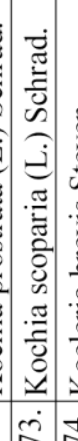 & 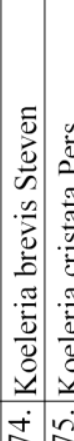 & 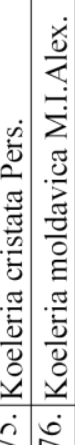 & 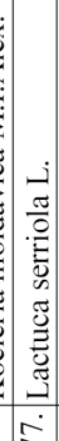 & 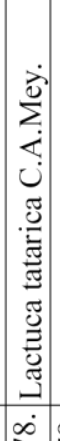 & 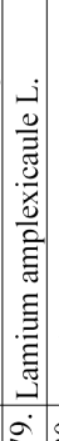 & 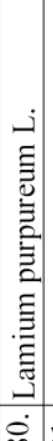 & 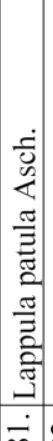 & 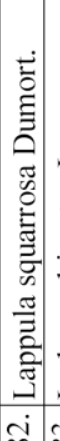 & 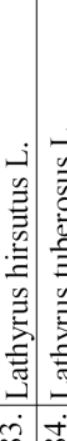 & 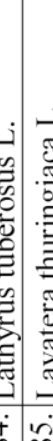 & 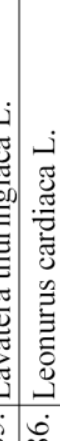 & 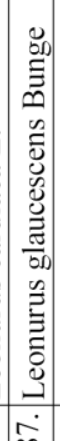 & 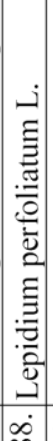 & 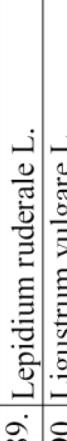 & 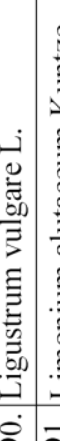 & 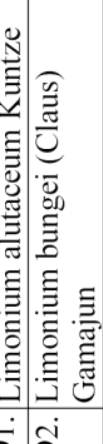 & 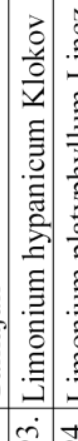 & 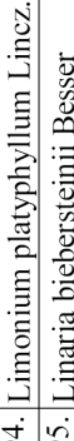 & 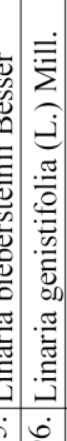 & 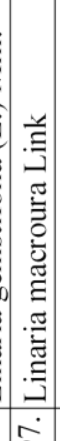 & 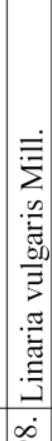 \\
\hline & & & & & & & & & & & & & & & & & & & & & & & & & \\
\hline
\end{tabular}




\begin{tabular}{|c|c|c|c|c|c|c|c|c|c|c|c|c|c|c|c|c|c|c|c|c|c|c|c|c|c|}
\hline$\stackrel{2}{*}$ & $=0$ & $a$ & $a$ & $\sim$ & 2 & $<<$ & $<$ & $a$ & 2 & $2=$ & a & $=5$ & $\sum_{4}^{\infty}=$ & $=$ & 2 & $<$ & $<$ & 2 & $\sum_{<}^{n}$ & & 2 & $\sum_{<}^{\infty}$ & 2 & $\infty$ & $\frac{n}{<}<$ \\
\hline 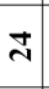 & $=-$ & & $=\overleftrightarrow{\Xi}$ & $=$ & $\Xi$ &.- & -5 & $\begin{array}{l}\text { की } \\
\text { of } \\
=\end{array}$ & on & $\sum_{\Sigma}^{\mathscr{E}}$ & $=$ & $\overleftrightarrow{\Xi}$ & 5 & $5=$ & $=$ & - & - & $=$. & $\Xi$. & -5 & $==$ & $=$ & $=$. & $\sqsubseteq$ & $E-$ \\
\hline$\approx$ & $\tilde{z}$ & 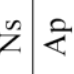 & $\vec{z}$ & $\stackrel{\Xi}{\Xi}$ & $\dot{\xi}$ & $\begin{array}{l}\infty \\
0\end{array}$ & दे & $z^{n}$ & $z^{n}$ & $\bar{z}$ & $z^{n}$ & $\begin{array}{l}\infty 0.1 \\
1\end{array}$ & 女 & $\dot{z}$ & $\cong$ & < & $z$ & $\cong$. & \&े? & $z$ & $\ddot{\Delta}$ & $\stackrel{2}{<}$ & $z$ & \& & $\frac{n}{z}$ \\
\hline$\approx$ & $\cong$ & 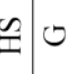 & 0 & 0 & ن & ن & ט & 0 & 0 & 0 & ט & 0 & ن & 0 & 0 & 0 & 里 & 0. & 0 & $\mathscr{E}$ & 0 & 0 & 牛 & ט & 0 \\
\hline $\bar{\sim}$ & \pm- & -- & -+ & $\sim$ & + & - & 0 & - & - & $m$. & - & 0 & $->$ & $N$ & $=$ & - & $\infty$ & $\infty$ & $\wedge:$ & $m$ & in & 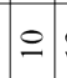 & $\cong$. & + & \pm 0 \\
\hline సิ & & & & & - & & & & & & & & & & - & & &.- & - & $\mathrm{N}$ & & & & & -- \\
\hline 2 & $\mathrm{~N}$ & & $m$ & & & & & & & & & - & - & -- & $m$ & & & N. & - & & - & - & $N$ & & \\
\hline$\stackrel{\infty}{\infty}$ & $\sim$ & - & -- & - & & & - & & & & & & & - & -4 & & - & $\sim$. & - & $\sim$ & & - & $N$ & & $-a$ \\
\hline 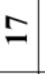 & & & & & & & - & & & & & - & & & $\sim$ & & & $m$ & & & $-N$ & - & 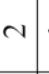 & - & - \\
\hline 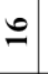 & & & & & & - & & & & & & & & & $N$ & & - & $N$ & & $N$ & - & & - & & -4 \\
\hline$\because$ & $m$ & & & & & & & & & - & & - & - & & - & & & - & & $m$ & & & & - & $4 d$ \\
\hline \pm & & & & & & & & & & & & & & - & -- & & & - & & $N$ & & - & & & 4 \\
\hline$\cong$ & & & $\sim$ & & & & & & & & & & & & $\sim$ & & & $N$ & & & - & $N$ & $N$ & & $\mathrm{~N}$ \\
\hline $\mathcal{I}$ & & & & & & & & & & & & & & $r$ & $N$ & & - & $\sim$ & & & & & & & $N$ \\
\hline$=$ & & & & & & & & & & & & & & $r$ & 10 & & - & $\sim$. & $-c$ & $N$ & & & $N$ & & v \\
\hline$\varrho$ & & & & & & & & & - & - & - & - & & & $m$ & & & $m$ & & $N$ & N & $N$ & $m$ & $N$ & \\
\hline$a$ & & & & - & - & & & & & & & & & - & $-N$ & & - & $\sim$ & & $m$ & & & $N$ & & $=-$ \\
\hline$\infty$ & & & & & $N$ & & - & & & & & & & & & & & $\sim$ & $N$ & $N-$ & & & - & & - \\
\hline r & - & & $N$ & & & & & & & & & & & - & N & & - & $\sim$ & - & $m$ & & N & - & & v \\
\hline 6 & & & & & & & & & & & & & & & $N$ & & - & $m$ & & $N$ & & $\sim$ & $N$ & & \\
\hline no & & & & & - & & - & - & & - & & - & & & - & & & $N$ & & $m$ & & & & & N \\
\hline+ & & & & & & & - & & & & & - & & & $\sim$ & & & $\sim$ & & & & $N$ & $m$ & & - \\
\hline " & - & & & & & & - & & & & & & & r & $v-$ & -1 & - & - & - & $m$ & & - & - & - & - \\
\hline
\end{tabular}

m
m
m
-1
-1
-
-1
-

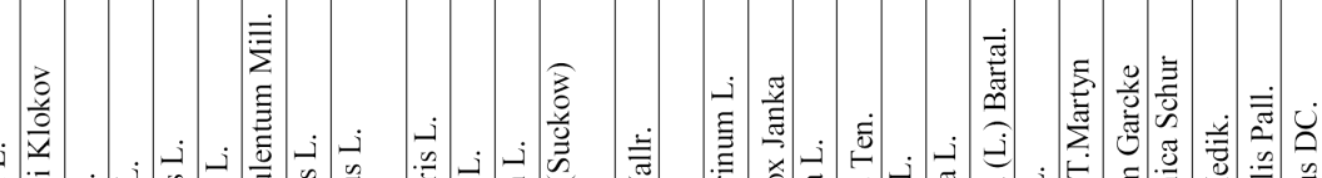

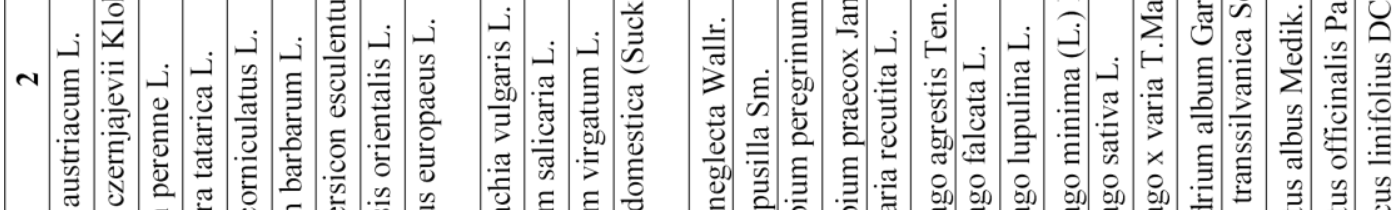

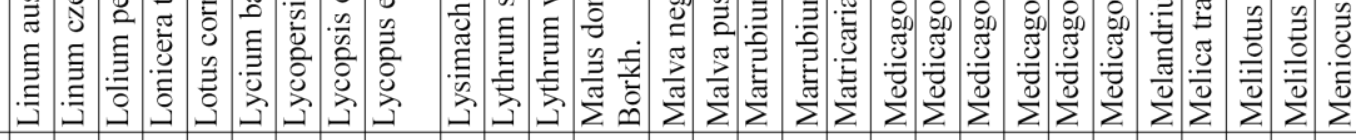

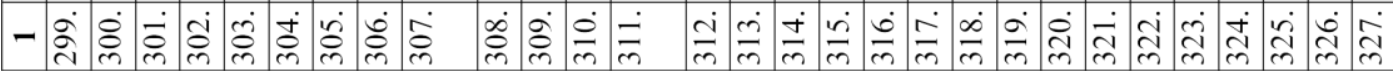


Moysiyenko I.I., Dayneko P.M., Sudnik-Wójcikowska B., Dembicz I., Zachwatowicz M., Zakharova M.Ya.

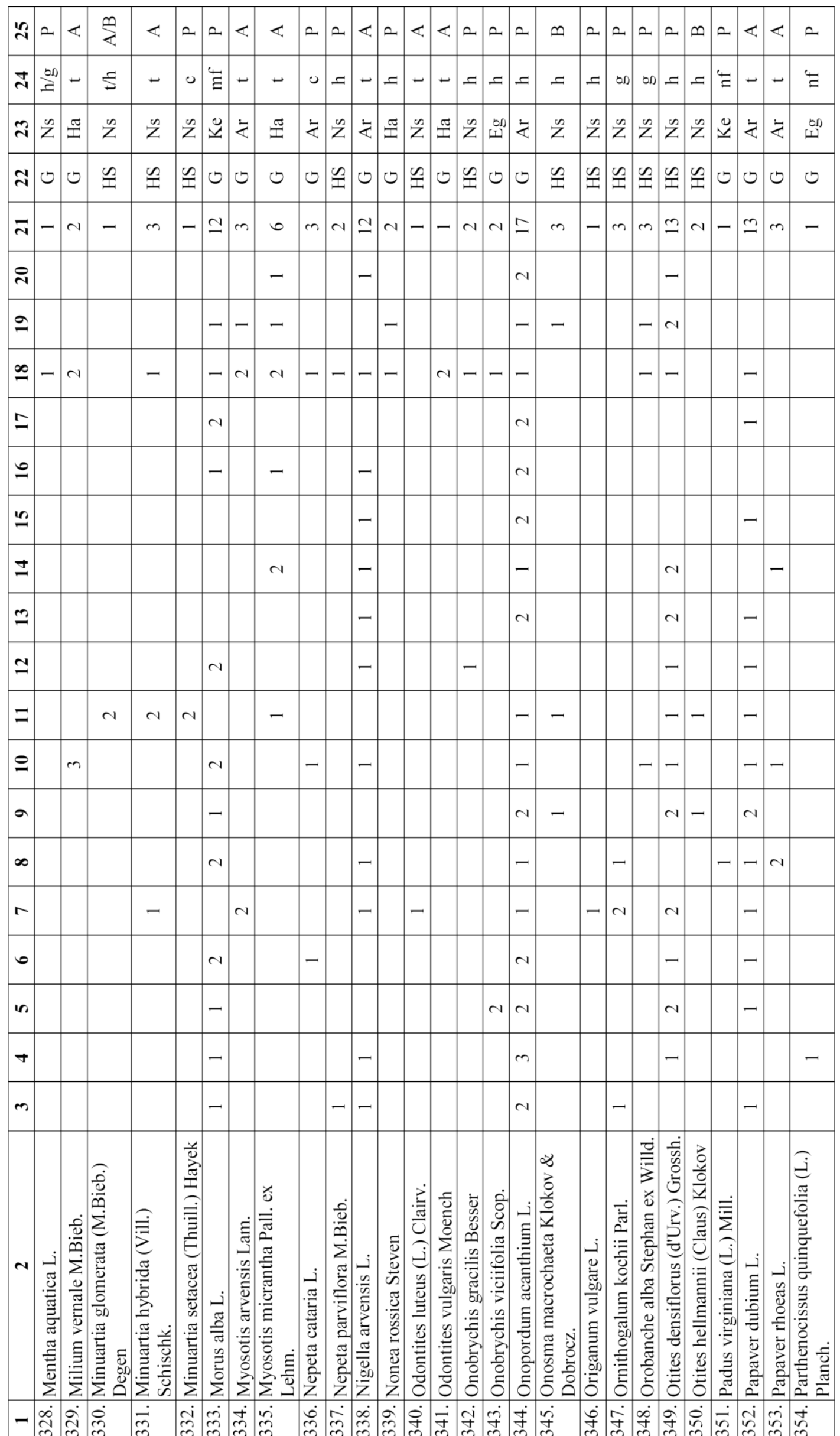




\begin{tabular}{|c|c|c|c|c|c|c|c|c|c|c|c|c|c|c|c|c|c|c|c|c|c|c|c|c|c|}
\hline 2 & $=2$ & $2<<$ & 2 & $=$ & 28 & $=0$ & 20 & 20 & $4=$ & 2 & 2 & 2 & $=$ & $2=$ & 2 & $<1$ & $2=$ & $2=$ & $<$ & $<$ & $\ll$ & 2 & $<$ & 2. & 2 \\
\hline $\mathbf{N}$. & $=\stackrel{0}{I}$ & $\begin{array}{l}0 \\
\text { L. }\end{array}$ & מם & as & $=-$ & $=-$ & & Do 0 & $=$ & $=$ & $=$ & $=$ & $=$. & $=-$ & $=$ & $-\quad \stackrel{0}{=}$ & $\stackrel{5}{ \pm 01}$ & $\stackrel{\infty}{=} \stackrel{\infty}{=} \stackrel{0}{=}$ & - & - & - & $\overleftrightarrow{\Xi}$ & - & & $=$ \\
\hline$\tilde{\imath}$ & $\tilde{z}: \ddot{z}$ & $\ddot{v})$ & $z^{n}$ & $z^{n}$ & & & & $\hat{z}$ & 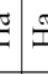 & $\cong$ & $z$ & $z$ & $\stackrel{\Xi}{=}$. & दे & $\stackrel{\Xi}{ \pm}$ & $z^{n}$ & $\cong \cong$ & $\stackrel{\Xi}{\Psi} \stackrel{\Xi}{\Psi}$ & $\ddot{\underline{u}}$ & दे & $z^{n}$ & $z^{n}$ & $\dot{z}$ & $\mathbb{\pi}$ & $z$ \\
\hline ה & $\cong 0$ & ט & $\cong$ & $\cong$ & $\cong$ & & $E \cong$ & $E$ & 0 & 0 & $\cong$ & 0 & 0 & 0 & $\cong$ & $\cong$ & 0 & 0 & 0 & 0 & 0 & U & ن & & $\cong$ \\
\hline $\bar{\sim}$ & $+\curvearrowright$ & $\sim-$ & $m$ & - & +0 & $\sqrt{N} \propto$ & $\circ \cong$ & $=r$ & $\infty$ & 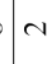 & - & - & $\simeq$. & -4 & in & 0 & $\stackrel{\infty}{\infty}$ & $\infty m$ & - & 0 & a & - & a & $\because=$ & \pm \\
\hline 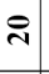 & & & & & & & & 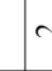 & & & & & $\sim$ & & & & $m m$ & $\therefore-$ & & & & & & $-c$ & $\mathrm{~N}$ \\
\hline 2 & & & & - & & $v$ & - & & & - & & & $\sim$ & & $\sim$ & & $m m$ & $n$ & - & & & & & & 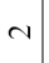 \\
\hline$\stackrel{\infty}{\infty}$ & $N$ & - & & & & Y & $v o$ & $1-$ & - & & & - & - & & - & $-m$ & $m$ & $n$ & & - & & & - & $-c$ & $N$ \\
\hline$\simeq$ & & & & & & & - & & r & - & - & & $\sim$ & & & & $m m$ & $?$ & & $N$ & & & - & & - \\
\hline$\simeq$ & & & - & & & & - & - & 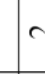 & & & & $N$. & -- & - & & $m$ & $n$ & & & & & - & $-\Omega$ & 4 \\
\hline$\because$ & & & & & & & & - & - & & & & & & & & $m m$ & $n$ & & & & & - & & $m$ \\
\hline$\Xi$ & & & & & $\sim$ & r & $v-$ & & & & & & & & & \begin{tabular}{l|l}
$N$ & $N$
\end{tabular} & $\sim m$ & $n$ & & & & & & $N$ & \\
\hline 2 & & & & & & & & & & & & & $N$ & & & & $m m$ & $n$ & & & & & & & - \\
\hline$\simeq$ & & & & & & - & & & & & & & $N$ & & & \begin{tabular}{l|l}
$n$ & $m$
\end{tabular} & $m m$ & $=-$ & & & & & $N$ & & $\sim$ \\
\hline$=$ & & & - & & - & & & & & & & & - & & $\sim$ & & $m A$ & v & & & & & - & $-c$ & $N$ \\
\hline$=$ & $-N$ & v & & & & - & $-\gamma$ & N & - & & & & & & & & $c A$ & v - & & & & & & & $N$ \\
\hline$a$ & & & & & & & - & & r & & & & $N$ & & -7 & $-d$ & $\sim m$ & $?$ & & $\sim$ & & - & $N$ & & $\sim$ \\
\hline$\infty$ & & v & & & & & & - & -1 & & & & $N$ & & & & $m m$ & $?$ & & & & & & & \\
\hline$r$ & $N$ & & & & - & ल & 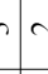 & & & & & & & & & $n$ & $m m$ & $n$ & & - & - & & - & & - \\
\hline 0 & & & & & & - & - & - & & & & & & & & & & $n$ & & & & & & & - \\
\hline in & & & & & & & - & $-\gamma$ & $1-$ & & & & & & & & $N m$ & $n$ & & & & & & & - \\
\hline 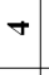 & & & & & & ल & - & & & & & & - & & & & $m \sim$ & $v$ & & $N$ & & & & - & \\
\hline$m$ & $N$ & & - & & - & r & & & & & & & $N$ & & & $-N$ & $N m$ & $n$ & & $N$ & - & & $N$ & - & \\
\hline$\sim$ & 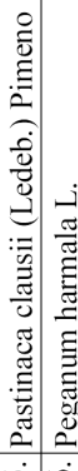 & 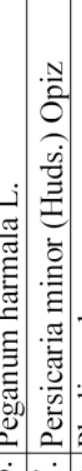 & 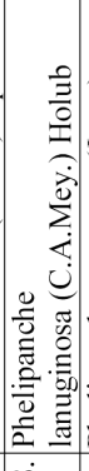 & 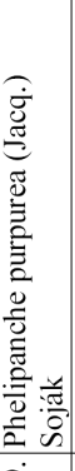 & 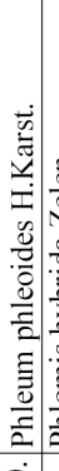 & 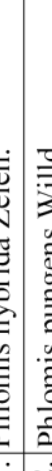 & 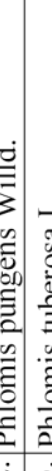 & 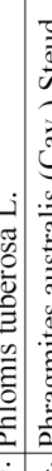 & 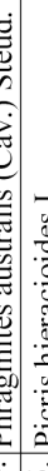 & 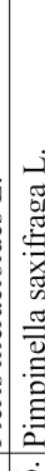 & 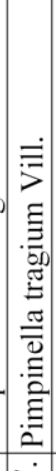 & 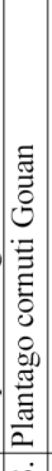 & 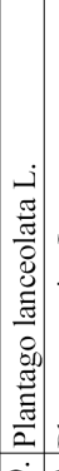 & 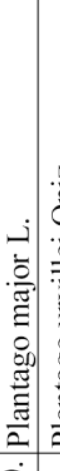 & 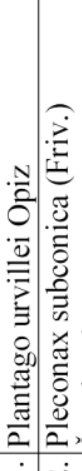 & 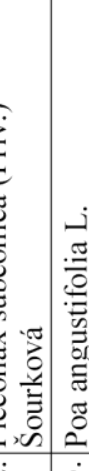 & 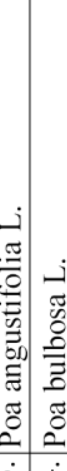 & 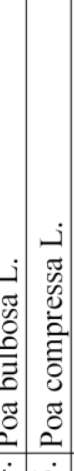 & 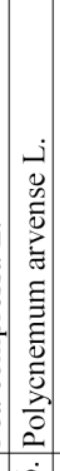 & 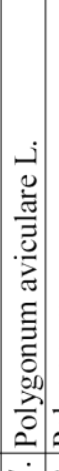 & 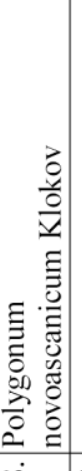 & 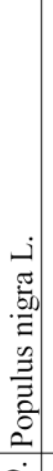 & 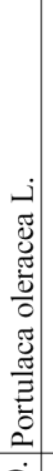 & 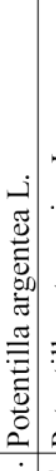 & 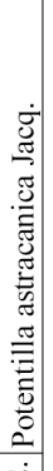 \\
\hline & $\begin{array}{l}n \\
m \\
m\end{array}$ & & & & & & & & & & $\begin{array}{l}\dot{0} \\
0 \\
\end{array}$ & $\left|\begin{array}{c}0 \\
0 \\
0 \\
\end{array}\right|$ & & & & i & $n$ in & $\begin{array}{c}\dot{y} \\
n\end{array}$ & & & & & $\begin{array}{l}\dot{\infty} \\
\substack{\infty \\
m}\end{array}$ & \begin{tabular}{c}
$\dot{\infty}$ \\
$\substack{\infty \\
\hdashline}$
\end{tabular} & $\begin{array}{c}i \\
\infty \\
\infty \\
\end{array}$ \\
\hline
\end{tabular}


Moysiyenko I.I., Dayneko P.M., Sudnik-Wójcikowska B., Dembicz I., Zachwatowicz M., Zakharova M.Ya.

\begin{tabular}{|c|c|c|c|c|c|c|c|c|c|c|c|c|c|c|c|c|c|c|c|c|c|c|c|c|c|}
\hline $\mathbb{A}$ & $a$ & 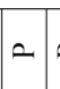 & $\sim=$ & $\omega$ & $a$ & $a$ & 2 & 2 & $\varangle$ & $a$ & 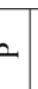 & 2 & $a$ & & $a=$ & 0 & 2 & 2 & $2=$ & $=0$ & 2 & & $=2=$ & & $\stackrel{\vec{n}}{\frac{\partial}{n}}$ \\
\hline$\vec{N}$ & $=$ & $=$. & $=$ & $=$ & $=$ & $=$ & $\vec{\Xi}$ & $\Xi$ & - & $\Xi$ & $\Xi$ & . & $\stackrel{\text { on }}{\geq}$ & $\tilde{s}$ & $\Xi$ & $\exists$ & $=$ & $\vec{\Xi}$ & $\vec{\Xi}=$ & $=4$ & $=$ & 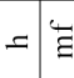 & $\bar{E} \mid$ & & $=$ \\
\hline$\vec{\sim}$ & $z^{n}$ & $\dot{z}$, & $\tilde{z}$ & $z$ & $z^{n}$ & $z^{n}$ & 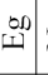 & $z^{n}$ & $\Xi$ & 年 & $\stackrel{\pi}{ \pm}$ & $z$ & $z^{n}$ & & $z \approx$ & $\ddot{v}$ & $z^{n}$ & $z^{n}$ & $z$ & $z \bar{z}$ & 妾 & & $\Xi$ & & z \\
\hline$\tilde{N}$ & $\cong$ & $\cong$ & 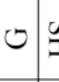 & 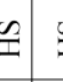 & $\cong$ & $\cong$ & ט & ט & ט & ט & 0 & $\cong$ & $\cong$ & \begin{tabular}{|c|c}
0 & 0
\end{tabular} & 0 0 & ט & $\cong$ & ט & 0 & 0 0 & 0 & ט & ט & ט & 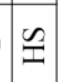 \\
\hline $\bar{N}$ & $\sim$ & 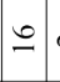 & $N=$ & \pm & in & $N$ & - & \pm & $\cong$ & $=$ & - & \pm & + & $=:$ & $\cong ح$ & $N$ in & 0 & $\stackrel{0}{-}$ & -- & $-m$ & $m$ & \pm 0 & $0-$ & + & $\cong$ \\
\hline$\vec{\sim}$ & & - & & 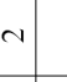 & & & & - & $N$ & & & $N$ & & & & - & & & & & & & & - & \\
\hline 2 & & $m$ & & $\sim$ & - & & & $\mathrm{N}$ & $\mathrm{N}$ & - & & & - & $N$. & - & 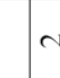 & & $N$ & - & & & -- & & & - \\
\hline$\infty$ & & $m$ & $N$ & & & & & $N$ & $N$ & $N$ & & - & & -1 & $N$ & & $\sim$ & $N$ & - & - & - & -- & - & & - \\
\hline I & & - & & & $N$ & & & $N$ & $N$ & - & & $N$ & & $\mathrm{~N}$ & $N$ & & - & - & & - & & $N \mid N$ & & & - \\
\hline$\underline{\varrho}$ & & & & - & & & & & $N$ & & & - & & & - & & & - & & & & - & & & \\
\hline 2 & & $m$ & & & & & & & $N$ & & & $N$ & & & & & & & & & & - & & & \\
\hline \pm & & $N$ & & - & & - & & $N$ & $m$ & & & $N$ & & & - & & & - & & & - & $N$ & & - & \\
\hline 2 & - & $\sim$ & & N & $m$ & & & $N$ & $N$ & $N$ & & & - & -0 & $\sim$ & $\sim$ & - & $N$ & & & & - & & & N \\
\hline$\simeq$ & & $N$ & & - & & & & - & $N$ & & & $N$ & &.- & - & & $N$ & $N$ & & & & & & & - \\
\hline$=$ & & $\sim$ & & $N$ & 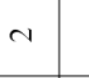 & & & - & $m$ & & & - & & - & -- & - & - & - & & & & $N$ & & & $N$ \\
\hline$\varrho$ & & $\sim$ & $N \Omega$ & $N$ & & & & $m$ & $N$ & $m$ & & $N$ & & - & $m-$ & - & - & $m$ & -- & $-r$ & $N$ & - & & & \\
\hline$a$ & & $\sim$ & & - & - & - & & $N$ & $N$ & & & $n$ & &.- & - & & & - & - & & & & & & 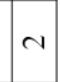 \\
\hline$\infty$ & & & & & & & & & $\mathrm{N}$ & - & & $N$ & & & & & & - & & & & -- & - & & - \\
\hline$r$ & & $\mathrm{~N}$ & & - & & & - & $\mathrm{N}$ & $m$ & $N$ & - & $\sim$ & - &.- & - & $r$ & & $\mathrm{~N}$ & - & & & & & & \\
\hline 0 & & N & & - & & & & - & $\mathrm{N}$ & $N$ & & & & - & & & & $N$ & & & & - & & & \\
\hline 10 & & $m$ & & - & & & & & $m$ & - & & $N$ & - & & & & & - & & & & $N-$ & - & - & \\
\hline$\nabla$ & - & $\sim$ & & - & & & & $N$ & $N$ & $N$ & & & & - & $\sim$ & $m$ & & 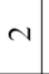 & - & & & $N$ & & & - \\
\hline$m$ & & $\sim$ & & - & & & & $\mathrm{N}$ & $m$ & - & & - & & & - & & & - & - & & & -- & & & - \\
\hline$N$ & 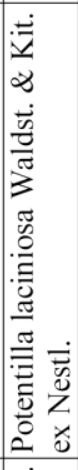 & 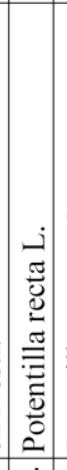 & 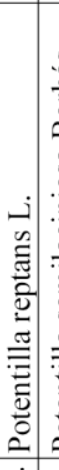 & 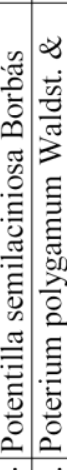 & 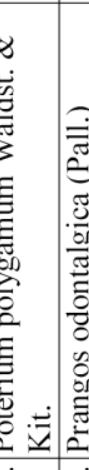 & 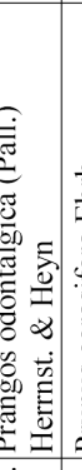 & 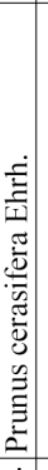 & 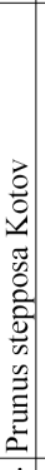 & 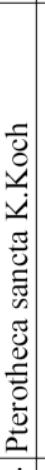 & 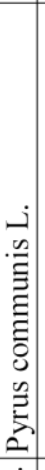 & 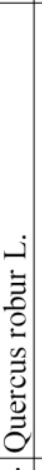 & 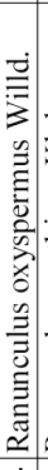 & 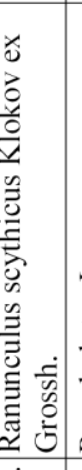 & 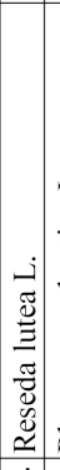 & 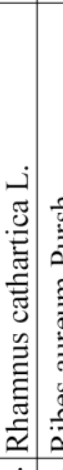 & 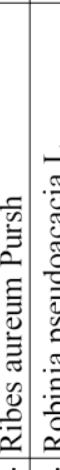 & 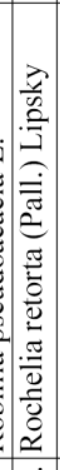 & 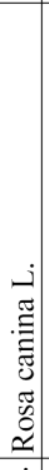 & 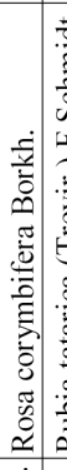 & 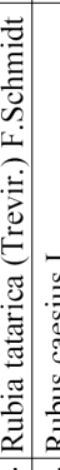 & 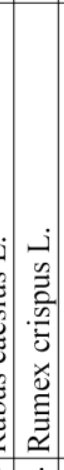 & 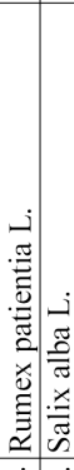 & 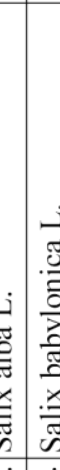 & 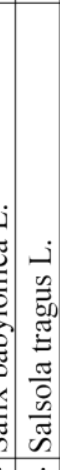 & 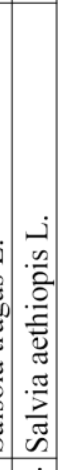 \\
\hline & & 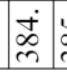 & \begin{tabular}{c|c}
$\dot{\infty}$ & $\substack{\infty \\
\infty}$ \\
\hdashline & $\infty$
\end{tabular} & 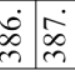 & \begin{tabular}{l|l}
$\dot{0}$ & 0 \\
0 & 0 \\
$n$
\end{tabular} & 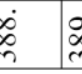 & & & & $\hat{c}$ & & & & \begin{tabular}{c|c}
$\dot{0}$ \\
$\dot{m}$
\end{tabular} & \begin{tabular}{l|c}
$\hat{c}$ \\
$\dot{c}$
\end{tabular} & 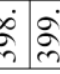 & $\begin{array}{l}\dot{8} \\
\dot{q}\end{array}$ & $\dot{\dot{o}}$ & \begin{tabular}{l|l}
$\dot{\sigma}$ \\
$\dot{\sigma}$
\end{tabular} & \begin{tabular}{l|l}
$\dot{b}$ \\
$\dot{b}$
\end{tabular} & $\begin{array}{l}\dot{1} \\
\dot{a} \\
\dot{y}\end{array}$ & \begin{tabular}{l|l} 
\\
$\stackrel{9}{9}$
\end{tabular} & & $\dot{8}$ & $\frac{\dot{\theta}}{7}$ \\
\hline
\end{tabular}




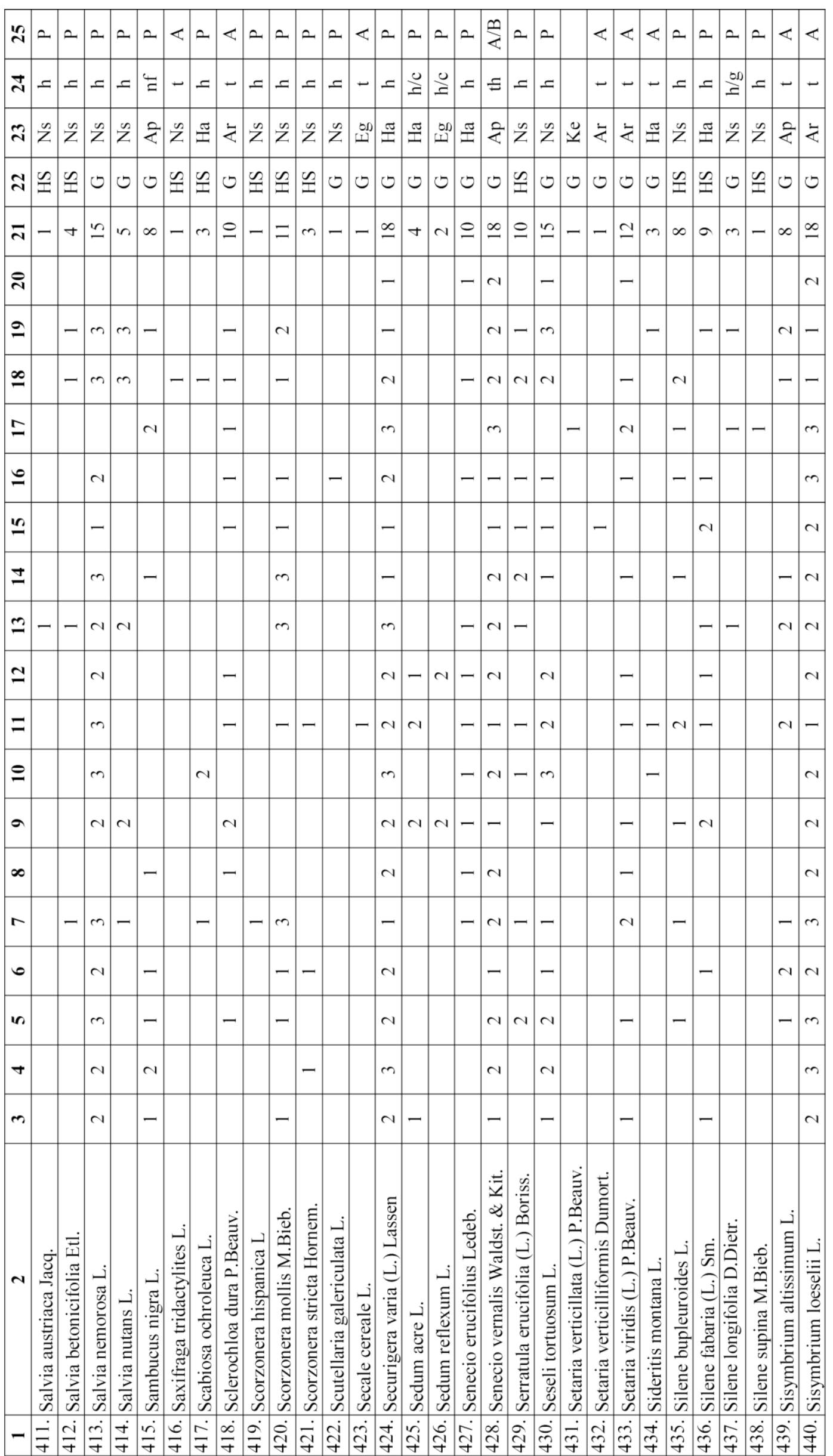


Moysiyenko I.I., Dayneko P.M., Sudnik-Wójcikowska B., Dembicz I., Zachwatowicz M., Zakharova M.Ya.

\begin{tabular}{|c|c|c|c|c|c|c|c|c|c|c|c|c|c|c|c|c|c|c|c|c|c|c|c|c|}
\hline $\mathscr{*}$ & 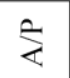 & 2 & $<8$ & $2<$ & $<$ & 2 & 2 & $\overbrace{<}^{\infty}$ & 2 & 2 & 2 & 2 & 2 & $=2$ & $a$ & $\sim=$ & $2=$ & $2=$ & 2 & 2 & 2 & $\stackrel{\vec{c}}{\infty}$. & $\ll$ & $\ll$ \\
\hline$\sqrt{2}$ & $\xi$ & 0 & - & $=-$ & - & 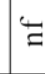 & $=$ & 5 & $=$ & $=$ & $=$ & $=$ & $\exists$ & $\stackrel{\text { on }}{=}=$ & $=$ & $=$ & $=0$ & $\begin{array}{lll}0 & 0\end{array}$ & 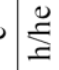 & 0 & $=$ & $=$ & - & - \\
\hline$\pi$ & $z^{n}$ & $\stackrel{\Xi}{=}$ & $\dot{4}$ & दे & 广 & $z_{z}^{n}$ & z & दे & $z$ & $z$ & z & z & $\stackrel{0}{\simeq}$ & \begin{tabular}{l|l}
$z$ & $\Xi$ \\
$z$
\end{tabular} & $z^{n}$ & से & $\Xi$ & \begin{tabular}{l|l}
$n$ & $n$
\end{tabular} & $z$ & $z$ & $z$ & 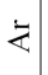 & 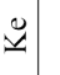 & $\dot{z}$ \\
\hline $\mathbb{N}$ & $\bullet$ & 0 & 0 & 0 & 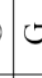 & en & 0 & ט & $\cong$ & 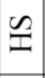 & $\cong$ & $\cong$ & 0 & $\cong$ & $\cong$ & 0 & $\cong 0$ & 0 & 0 & $\cong$ & ن & ט & ט & 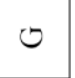 \\
\hline$\vec{\sim}$ & $\simeq$ & - & $\therefore-$ & $-r$ & - & - & r & $\simeq$ & \pm & in & - & N & + & $\cong N$ & \pm & in 1 & $r$ & $\wedge \simeq$ & 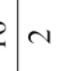 & $\simeq$ & $r$ & - & $\infty$ & $N$ \\
\hline స్ & & & & & & & & - & & & & & & & $N$ & -- & - & $N$ & & & & & & \\
\hline 2 & - & & & & & & N & - & $m$ & $\mathrm{~N}$ & & & & $m$ & & & -- & $-m$ & & & - & & $N$ & \\
\hline$\stackrel{\infty}{\infty}$ & $\sim$ & & - & & & & - & - & $N$ & - & & - & & $\mathrm{N}$ & - & - & & $-N$ & - & $N$ & - & & $\sim$ & \\
\hline$\Xi$ & & & - & - & & & & $m$ & & & & & - & $N$ & $N$ & & - & - & & $m$ & & & & \\
\hline$\simeq$ & & & & - & & & & N & N & & & & & & $N$ & - & & - & & & & - & & \\
\hline 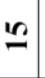 & $\sim$ & - & & - & & & & & $m$ & & & & & $m$ & $N$ & & - & - & & N & & & & \\
\hline \pm & $N$ & & & & & & & - & $\mathrm{N}$ & & & & - & $m$ & - & & & - & & 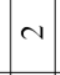 & & & & \\
\hline 2 & - & & & & & & N & & $m$ & - & & & & $N$ & & & $->$ & $N \mid N$ & & $N$ & - & & & \\
\hline$\simeq$ & & & & & & & & N & N & & & & & $N$ & $N$ & & & $N$ & & & - & & & \\
\hline$=$ & - & & - & & & & & & - & - & & & - & & - & & -1 & $N-$ & & N & & & - & \\
\hline$\varrho$ & - & & & & & & & N & $m$ & & & & - & $m$ & $m$ & & & $\mathrm{~N}$ & - & $m$ & a & & $m$ & \\
\hline$a$ & $N$ & & & & & & - & - & & & & & & & $m$ & - & & $N \mid N$ & & & - & & & - \\
\hline$\infty$ & & & & & & & - & $N$ & $m$ & & & & & & $N$ & & - & & & & & & & \\
\hline$r$ & $N$ & & & & - & $N$ & - & - & N & - & - & $N$ & & $N$ & - & & & -- & & $\sim$ & - & & $N$ & \\
\hline 0 & & & - & & & & & & $m$ & & & & & - & & & & 4 & & N & & & & \\
\hline in & $N$ & & & & & & & & $N$ & & & & & $N$ & - & & & $N$ & & $N$ & & & & 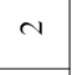 \\
\hline$\nabla$ & - & & & & & & - & & $\mathrm{N}$ & & & & & $N$ & & - & & - & & 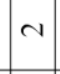 & & & & \\
\hline$m$ & $N$ & & - & & & & & - & & & & & & s & v & & & - & & & & & - & \\
\hline$\sim$ & 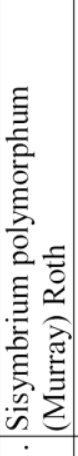 & 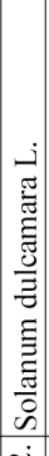 & 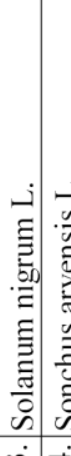 & 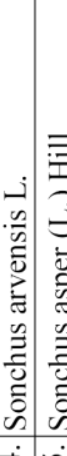 & 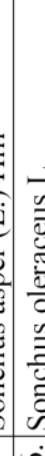 & & 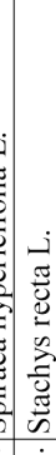 & 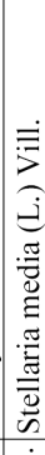 & 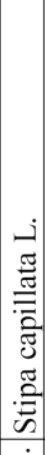 & 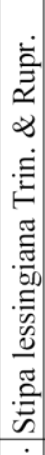 & 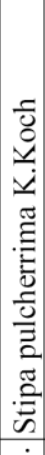 & 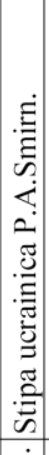 & 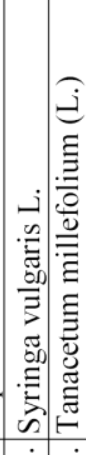 & 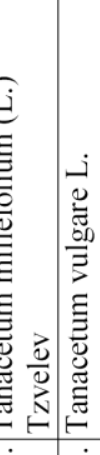 & 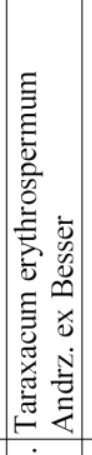 & 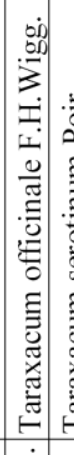 & 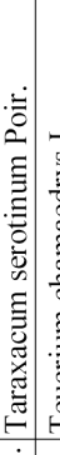 & 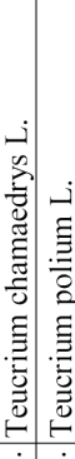 & 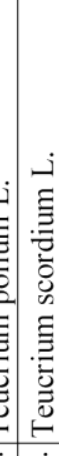 & 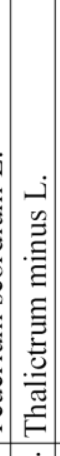 & 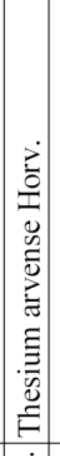 & 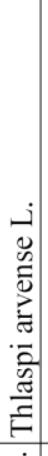 & 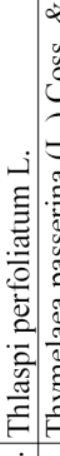 & 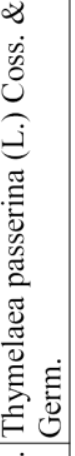 \\
\hline & & & $\begin{array}{l}\text { 守 } \\
\end{array}$ & & & & & & & & & & & & is & \begin{tabular}{l|l}
$\infty$ & 8 \\
$i n$ & 8 \\
\end{tabular} & 8 & \begin{tabular}{l|l}
$\dot{8}$ & $\dot{0}$ \\
& 0
\end{tabular} & రु & 触 & $\mid$ & & & \\
\hline
\end{tabular}




\begin{tabular}{|c|c|c|c|c|c|c|c|c|c|c|c|c|c|c|c|c|c|c|c|c|c|c|c|c|c|c|}
\hline 'a & 2 & 2 & $\sum_{<}^{\infty}$ & 2 & $<$ & $<$ & $\ll$ & $<=$ & 2 & $<$ & 2 & 2 & $\stackrel{2}{\infty}$ & 2 & 2 & 2. & 2 & $2=$ & $a=$ & $<$ & $\ll$ & $a$ & $\sim$ & $=2$ & $a$ & $a$ \\
\hline N & 0 & 0 & 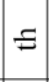 & $=$ & - & - & - & -5 & $=-$ & - & on & on & $=$ & $\stackrel{00}{I}$ & $\vec{\Xi}$ & $\Xi$ & 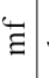 & $=-$ & $=0$ & D. - & - & $=$ & $=$ & $==$ & $=$ & $=$ \\
\hline$\vec{\sim}$ & $z^{n}$ & $z$ & दे & $\stackrel{2}{<}$ & $\stackrel{\otimes}{\simeq}$. & दे & $\Xi$ & z) & 4 & z & $z^{n}$ & 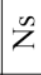 & $z^{n}$ & 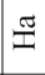 & $\Xi$ & $\Xi$ & 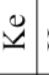 & 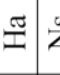 & $z^{n} \quad \tilde{z}$ & $\stackrel{\pi}{=}$ & $\ddot{z}$ & $z^{n}$ & $\stackrel{\Xi}{ \pm}$ & $\stackrel{\Xi}{\Xi}$ & $z$ & $\tilde{z}$ \\
\hline $\mathbf{N}$ & $\cong$ & $\cong$ & 0 & 0 & 0 & 0 & 0. & 0 & 0 & 0 & $\cong$ & $\cong$ & $\cup$ & ט & ט & ט. & ט & ن & ט & 0 & 0 & $\cong$ & 0 & ט ن & 0 & $\mathscr{E}$ \\
\hline $\bar{N}$ & $\stackrel{0}{\circ}$ & 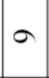 & in & $\stackrel{\infty}{\sim}$ & $\sigma$ & I & $-c$ & $m-$ & I & $\cong$ & $N$ & $N$ & - & - & $m$ & $\mathrm{~N}$ & $r$ & 6. & $-\infty$ & $=$ & in & $m$ & + & $\infty 0$ & $\infty$ & - \\
\hline$\tilde{\sim}$ & $\mathrm{N}$ & & &.- & - & - & & & & 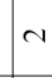 & & & & & & & & & $m$ & $m$ & & & & & & \\
\hline 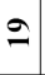 & $m$ & & & - & & N & & & & - & & & & & & & - & - & & & & & - & $-a$ & $\alpha$ & $\sim$ \\
\hline$\stackrel{\infty}{\infty}$ & 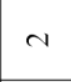 & & & - & & - & & & - & 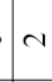 & $N$ & & & & $N$ & - & $\mathrm{N}$ & & $N A$ & $\mathrm{~m}$ & $N$ & & - & $\sim-$ & $\mathrm{N}$ & \\
\hline 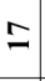 & $m$ & - & & $-c$ & $N$ & & & & ${ }^{\prime}$ & & & & & $N$ & & & & $N$ & & & & - & & - & $N$ & \\
\hline$\simeq$ & - & N & &.- & - & $\mathrm{N}$ & & & N & 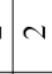 & & & & & & & $N$ & & & - & & & & & 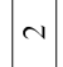 & \\
\hline 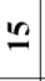 & - & & & $-c$ & $N$ & - & & & & $m$ & & & & & & & & & N & - & & & & & 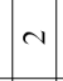 & \\
\hline \pm & & & & - & & $m$ & $-c$ & $N$ & & $m$ & & - & & & & & & - & $m$ & 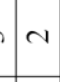 & & - & & - & 4 & \\
\hline 2 & m & - & & $N$ & & & & & - & & & & & & & & $\sim$ & & & & $\alpha$ & & & & 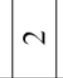 & \\
\hline $\mathbf{\simeq}$ & $N$ & - & $N$ & $N$ & & & & & - & $m$ & & & & & & & $N$ & & & & $m$ & & & & N & \\
\hline$\Xi$ & $\sim$ & & - & N. & - & - & & & & 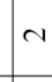 & & & & & - & & - & & & - & $\sim$ & - & & - & 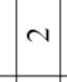 & \\
\hline$\varrho$ & & & & - & & & & & - & 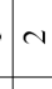 & & & & & $N$ & & & & & $m$ & & & - & \begin{tabular}{|c|c|}
4 & 4
\end{tabular} & - & \\
\hline$a$ & 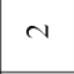 & - & - &.- &.- & - & & r & o & $m$ & & & & & & - & & & - & -2 & $N$ & & & $N-$ & $\mathrm{N}$ & \\
\hline$\infty$ & 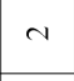 & - & - & $-c$ & $\sim$ & & & & $\sim$ & - & & & & & & & & $N$ & & & & & & - & $N$ & \\
\hline$r$ & - & N & & N. &.- & - & & $m$ & $m$ & $\alpha$ & - & N & - & & & & & & $\sim$ & 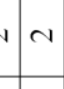 & & & & & $N$ & \\
\hline 0 & $N$ & & $\sim$ & $\mathrm{N}$ & & & & - & - & $N$ & & & & & & & - & $N$ & & & & & & - & - & \\
\hline 10 & $N$ & - & & $N$ & & - & & & & $\sim$ & & & & & & & & & - & - & & & & & $m$ & \\
\hline$\nabla$ & $\mathrm{N}$ & & & - & & - & & & $r$ & & & & & & & & & - & & & & & & & - & \\
\hline$m$ & - & N & & $-c$ & $\sim$. & - & & & c & - & & & & & & & & & - & $-\alpha$ & & & - & - & $\alpha$ & \\
\hline$\sim$ & 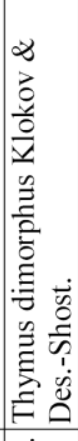 & 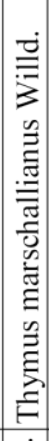 & 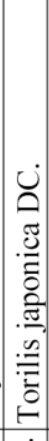 & 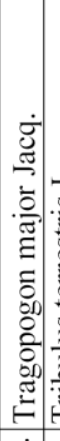 & 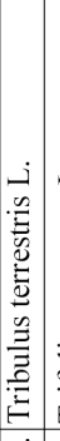 & . & 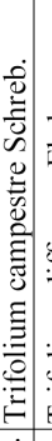 & ב & & 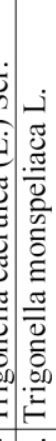 & 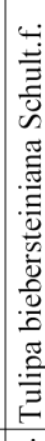 & 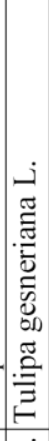 & $\begin{array}{l}\text { a } \\
\frac{\pi}{6}\end{array}$ & 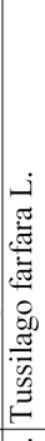 & 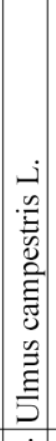 & 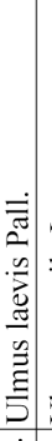 & 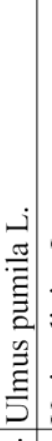 & ن. & 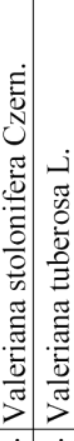 & 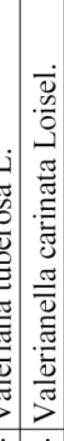 & 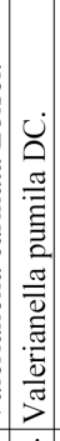 & 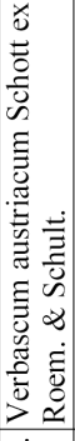 & 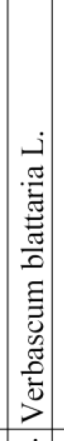 & 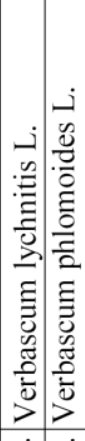 & 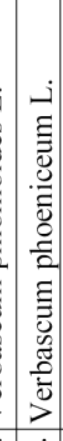 & 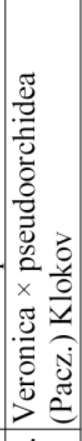 \\
\hline & \begin{tabular}{|l|}
0 \\
0 \\
+
\end{tabular} & 8 & 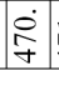 & $\begin{array}{l}\dot{8} \\
\dot{f}\end{array}$ & 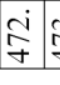 & 年 & 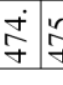 & 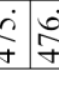 & & & & & & & & & & & \begin{tabular}{l|l}
$\dot{\infty}$ \\
$\dot{\alpha}$ \\
+
\end{tabular} & 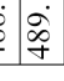 & & & $\mid \begin{array}{c}\dot{i} \\
\dot{q}\end{array}$ & 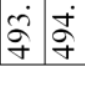 & & \\
\hline
\end{tabular}


Moysiyenko I.I., Dayneko P.M., Sudnik-Wójcikowska B., Dembicz I., Zachwatowicz M., Zakharova M.Ya.

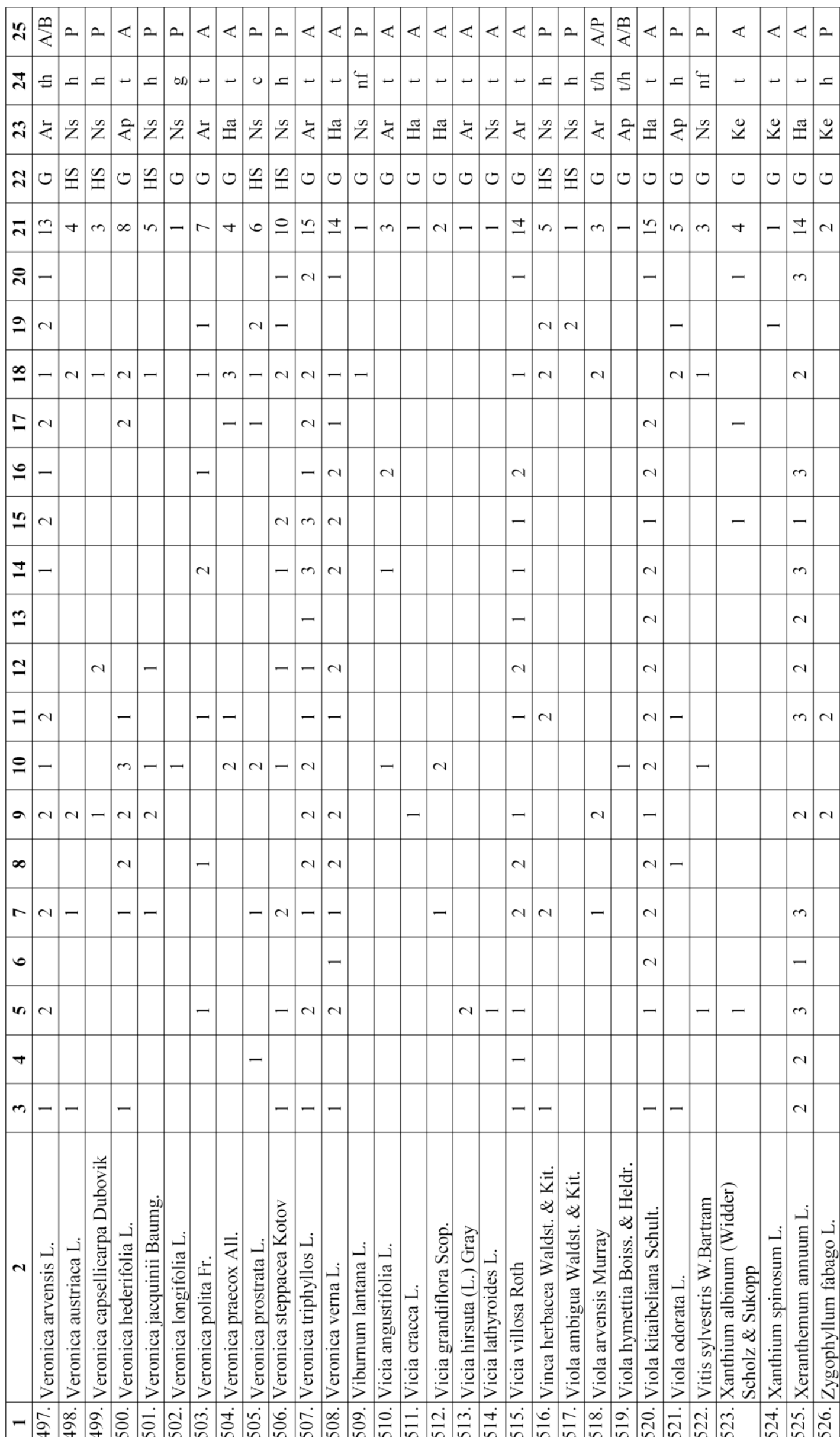

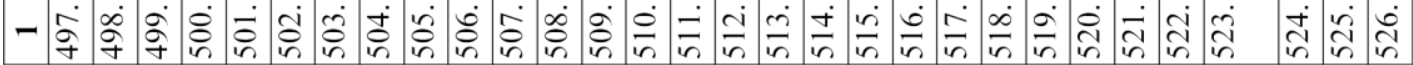


Abbreviations applied in Appendix 1:

Functional group:

$\mathrm{G}-$ generalists

HS - habitat specialists

Status in the Ukrainian flora:

Ns - non-synanthropic native species, not established in anthropogenic habitats;

Ap - euapophytes, natives established in anthropogenic habitats;

$\mathrm{Ha}$ - hemiapophytes, natives established only in semi-natural habitats;

Ek - ekiophytes, native species cultivated and escaped.

$\mathrm{Ar}$ - archaeophytes, aliens that immigrated before the year 1500;

$\mathrm{Ke}-$ kenophytes, aliens introduced after the year 1500;

Life forms:

$\mathrm{t}$ - therophytes;

th - short-living perennials $(2,3,4$ years life cicle);

$\mathrm{g}$ - geophytes;

$\mathrm{h}$ - hemicryptophytes;

hg - geophytes-hemicryptophytes - perennials, some of whose perennating buds (shootsystem) remain on the soil surface and underground;

hc - hemicryptophytes-chamaephytes - perennials whose perennating buds remain on orabove (within $0.25 \mathrm{~m}$ )

the soil surface;

c - chamaephytes;

$\mathrm{mf}$ - megaphanerophytes;

nf - nanophanerophytes;

Life span:

A - annual plant;

B - biannual;

$\mathrm{P}$ - perennials. 